\title{
Space and High Energy Experiments Advanced Electronic Systems 2012
}

\author{
Ryszard S. Romaniuk
}

\begin{abstract}
This paper is a research survey of the WILGA Symposium work. It presents a digest of technical effort results shown by young researchers from different universities during the Jubilee $\mathrm{XXX}^{\text {th }}$ SPIE-IEEE-Photonics Society of Poland Wilga 2012 symposium on Photonics and Internet Engineering. Topical tracks of the symposium embraced: nanomaterials and nanotechnologies for photonics, sensory and nonlinear optical fibers, object oriented design of hardware, photonic metrology, optoelectronics and photonics applications, photonics-electronics co-design, optoelectronic and electronic systems for astronomy and high energy physics experiments, JET tokamak and pi-ofthe sky experiments development. The symposium is an annual summary in the development of numerable Ph.D. theses carried out in this country in the area of advanced electronic and photonic systems. It is also a great occasion for SPIE, IEEE, OSA and PSP students to meet together in a large group spanning the whole country with guests from this part of Europe. A digest of Wilga references is presented [1]-[60]. This paper is the first part of the digest focused on astronomy, space, astroparticle physics, accelerators, and high energy physics experiments.
\end{abstract}

Keywords-Astronomy, optical observations, telescopes, space technology, accelerators, free electron laser, industrial standards for advanced electronics, photonic and electronic systems.

\section{INTRODUCTION}

$\mathbf{T}$ HE XXX ${ }^{\mathrm{TH}}$ JUBILEE SYMPOSIUM of young scientists WILGA 2012 on Photonics and Internet Engineering has gathered together over 300 participants in January and May Editions. There were presented over 250 papers - mainly concerning the realized Ph.D. theses and participation in research projects relevant to the topical area of the meeting. There were also presented a few plenary papers introducing the audience into new research areas of photonics and electronics. The symposium is organized under the auspices of SPIE - The International Society for Optical Engineering, IEEE (Poland Section and Region 8), Photonics Society of Poland, KEiT PAN, PKOpto SEP and WEiTI PW. The symposium is organized annually by young researchers from the PERG/ELHEP Laboratory of ISE PW with cooperation of SPIE and IEEE Student Branches. Media patronage over the symposium is extended by Elektronika monthly technical magazine. Symposium proceedings are published by Elektronika, IJET - Int. Journal of Electronics and Telecommunications by KEiT PAN and Proceedings SPIE. Wilga Symposium is topically associated with the cyclic research meetings on Optical Fibers and Their Applications organized in Białowieża (prof. J. Dorosz,

Ryszard S. Romaniuk is with Warsaw University of Technology, Institute of Electronic Systems, Nowowiejska 15/19, Warsaw, Poland (e-mail: rrom@ise.pw.edu.pl).
Białystok Univ. Technology) and in Nałęczów, (UMCS Univ., and Lublin Univ. Technology, prof. W. Wójcik) every 18 months. Below, there are presented some presentations from the main of the most interesting sessions or topical tracks of WILGA 2012 Symposium. Consecutive parts debate the sessions on Photonics Applications in Astronomy and Space Technologies; accelerator science; photon physics and plasma research; optoelectronics, biomedical and artificial intelligence.

\section{TOPICAL TRACKS OF WILGA 2012}

The topical session and tracks of WILGA 2012 were as follows: nanotechnologies and nanomaterials for optoelectronics and photonics, optical fibers for sensors and all-photonic devices for sensors, active optical fibers, sensors and sensory networks, object oriented design of optoelectronic and photonic hardware, photonics applications, advanced bioelectronics and bioinformatics, co-design of hybrid photonic - mechatronics and electronic systems, computational intelligence in optoelectronics and robotics, development in the wide-angle astronomic observations of the whole sky - pi-of-the-sky project, processing and imaging of multimedia data streams, machine vision, vehicles - quadrocopter and Mars rover, analog transmission systems in noisy conditions with digital reverse transmission channel, optoelectronic and photonic metrology, reconfigurable measurement systems, high performance - low-jitter low-latency transmission systems - White Rabbit, thermonuclear fusion experiments - JET and ITER, research results update from HEP experiments - TOTEM and CMS/LHC in CERN. A number of Wilga sessions concerned applications aspects of photonic and electronic circuits and systems, including in this advanced applications which combine hardware and software. A separate session track was organized by SPIE, IEEE, OSA and PSP - Photonics Society of Poland students for the new students beginning their adventure with the science of photonics and electronics.

\section{Astronomy and Space Technologies}

During the $\mathrm{XXX}^{\text {th }}$ Jubilee Symposium WILGA 2012 there were organized two topical sessions on observational astronomy (IR, optical and X-ray), space and satellite technologies, measurements and photonic/electronic equipment for these applications. The sessions were chaired by dr Piotr Orleański from CBK PAN (Space Research Center SRC PAS) Warsaw and prof. Filip A. Żarnecki of Faculty of Physics UW Warsaw in cooperation with prof. L. Mankiewicz of ZFT PAN and 
CAMK PAN (Mikołaj Kopernik Astronomical Center). The sessions gathered a few tens of young scientists and engineers from several research groups. There were presented over 20 papers concerning the current technical involvement of these labs. These sessions were organized a few months before Poland was going to be accepted as a new full member of the ESA - European Space Agency.

\section{Electronic AND Photonic EQUipMEnt For SATELLITES}

PICO and NANO SATELLITES: Picosatellites and nanosatellites cheaply invade the space for massive and varied research purposes. They are equipped in specialized miniature instruments optical, photonic, magnetic and electronic. The first part of the word satellite refers to their minute size. A satellite of the volume of 1 liter is usually called a picosatellite, while 10 liters satellite would be called nano. Why do astro researchers sent picosatellites to space? They are low cost. They carry pretty advanced scientific experiments. They create inexpensive platform for technology tests. They are initial platform for making bigger systems. They can form clusters while on the orbit, like proposed TechSat-21 mission. A picosatellite or a cluster of them can easily, and nearly at no cost, be added to larger space missions as the weight is usually lower than a few $\mathrm{kg}$ and power consumption less than a few W. CubeSat is one of proposed standards for a universal family of picosatellites. The international CubeSat project started in 1999. The aim was to provide a standard for design of picosatellites to reduce the cost and development time, increase accessibility to space and to sustain frequent launches. The standardized and open access CubeSat Design Specification largely simplifies building satellites. Small satellites frequently use common solutions and components for OBC (on-board computer) systems like ARM processors, TI secondary processors, PPC processors, embedded processors in FPGA fabric, single board computers, reconfigurable computers, standardized solutions for Mission Interface Computers - MIC. Most of these systems have multiple low-power modes of work. Some of them offer in-flight configuration of FPGA fabric. Typical I/O ports include servicing of telemetry, optical, magnetometry, thermal, communication, etc. Some of such systems are offered by: GOM Space, Clyde Space, Andrew Space, Space Micro, and more. The latter vendor designs the ProtonX-Box which is an Avionix Suite for CubeSats. It resides on a single board computer with PPC, DSP and FPGA chips.

The requirements for the OBC design are as follows: low power consumption, choice for multiple low-power modes, high computing power, resistance to radiation present at low Earth orbit, high reliability, low cost, small weight, board size suitable for picosatellite standard (like CubeSat), usage of common communication links as are often used in picosatellite designs, preparedness for standardized operating scenarios, fast communication links for payload and radio. A new architecture for OBC was chosen with ATmega128 microprocessor realizing tele-commands, telemetry, power control, logs and task queue. Spartan FPGA realizes operation of payload, processing, compressing and storing of payload data, and ADCS algorithms. Power consumption of this system is: active PC (at around $10 \mathrm{MHz}$ ) uses $100 \mathrm{~mW}$, idle PC uses $50 \mathrm{~mW}$, sleeping PC uses $100 \mu \mathrm{W}$. FPGA chip (at $100 \mathrm{MHz}$ ) uses below $2 \mathrm{~W}$. The peripherals of this system are: FLASH, ADC, RTC and RAM. The I/O ports are UART, USB, I2C, CAN, and high speed serial links. Payload, radio, PSU, ADCS units are using I2C. Test port is using USB and UART. Payload and radio are using HS serial links. Estimations for SEL and SEU effects in LEO for both micro-processors, are well beyond (i.e. one order of magnitude greater) the lifetime of the satellite. Reliability in the system is obtained by hardware redundancy.

BRITE (Bright Target Explorer) network of six satellites represents a new approach in space research. They are small, cheap, very specialized, and easy to be launched to the polar orbit. The constellation of six BRITE nanosatellites will continuously monitor (multi-spectrally in blue and in red) small changes in the brightness of nearby (i.e. very bright) hot stars, in order to study their oscillation behavior. Out of the six, two satellites Lem and Heweliusz will be made in Poland at CBK PAN. Such observations of the brightest stars cannot be made by large telescopes. Observation from the orbit avoids disturbances caused by Earth's atmosphere and adds paralax (simultaneous multiple and distant observation points to improve the imaging). The constellation of satellites assures the continuity of observations of chosen group of stars. The total mass of the nano satellite is $7 \mathrm{~kg}$ and it is of a cubical shape with $20 \mathrm{~cm}$ in dimension. The satellite consists of an optical telescope, computers (HKC, ACS, IOBC), communications, power system (control boards, PV cells, regulators), ACS magnetometer, star tracker, sun sensor, torquers, reaction wheels, radio receiver $(2.2 \mathrm{GHz})$ and transmitter $(437.365 \mathrm{MHz})$. The scientific payload done at CBK PAN is a red telescope, while the technological payload are: power supply unit, solar cells, battery, lock and release mechanism, micro-antenna boom, radiation dosemeter and beacon. The launch schedule of Polish Scientific Satellites is: BRITE-PL1 Lem - end of November 2012, BRITE-PL2 Heweliusz - second half of 2013. Additional payload is considered for Heweliusz. The objectives are tests of COTS photonic/electronic components and systems - candidates for long space missions by SRC. Details include: verification of LEO radiation simulations, RadFET and LED dosimetry, evaluation of fault mitigation techniques, components behavior studies including FLASH, SDRAM, ADC, FPGA. These tests are considered after the basic experiments are finished.

SPHINX: Sphinx X-ray solar spectrometer, constructed by CBK PAN in Wrocław is one of the instruments at the Coronas-Photon satellite. The satellite was launched in January 2009 from Plesetsk. The instrument weight was $4 \mathrm{~kg}$ and power consumption $10 \mathrm{~W}$. It detected photons in the range $1-15 \mathrm{keV}$, with time resolution $6 \mu \mathrm{s}$, energy resolution $500 \mathrm{eV}$. It provided telemetry data of $150 \mathrm{MB} /$ day. The scientific objectives of the experiment were: investigations of quiet corona heating processes via photon arrival time analysis; studies of short period soft X-ray oscillations; search for transient ionization plasma effects to determine flaring plasma densities, analysis of chemical composition of plasma (Al, $\mathrm{Ar}, \mathrm{Ca}, \mathrm{Fe}, \mathrm{Mg}, \mathrm{Si}, \mathrm{S}$ ); 
determination and time variability of differential emission measure (DEM); creating a reference photometric standard in soft X-rays with absolute accuracy of $10 \%$; verification of a novel, fluorescence based, photometry measurement method; monitoring of solar X-ray flux in the intensity range covering seven orders of magnitude. SphinX is comparable to similar orbiting instruments: Resik, GOES, SOXS, RHESSI. SphinX measures soft X-rays with four silicon PIN diode XR-100CR detectors, with different apertures to measure wide range of fluxes. The entrance windows of the detectors are covered with $13 \mu \mathrm{m}$ beryllium foil. Each detector has temperature sensor, Peltier cooler (operational temperature $-20^{\circ} \mathrm{C}$ ) and FET preamplifier inside the package. SphinX NG is under design. New types of detectors are used of SDD type (silicon drift detectors). They are characterized by much larger dynamic range than PIN diodes. CdTe detectors are also added. The measurements of photons are done in the range of $4 \mathrm{keV}$ to $200 \mathrm{keV}$. All the measurement system is fully digitized with filters, pulse height analyzer and controls implemented in FPGA.

ESA SOLAR ORBITER - STIX: The SRC PAS in Warsaw participates in the ESA Solar Orbiter (SO) mission with the STIX instrument. SO explores the Sun - heliosphere connection in the visible, extreme ultraviolet and X-rays. SO has elliptical orbit around the Sun with perihelion as low as $0.28 \mathrm{AU}$ (closer than ever before) and with increasing inclination up to $25^{\circ}$ with respect to the solar equator. Life time is predicted for 7 years nominal with launch time in 2017 (from Cape Canaveral), then cruising and reaching Sun orbit in 2021. Six gravity assists are scheduled (two by the Earths and four by Venus) to reach elliptical near Sun orbit - lasting for 168 days. STIX tries to answer the following questions: How and where do the solar wind plasma and magnetic field originate in the corona? How do solar transients drive heliospheric variability? How do solar eruptions produce energetic particle radiation that fills the heliosphere? How does the solar dynamo work and drive connections between the Sun and the heliosphere? The SO will try to determine in-situ the properties and dynamics of plasma, magnetic fields and particles in the near-Sun heliosphere, to survey the detail of the Sun's magnetized atmosphere, to identify the links between activity of the Sun's surface and the resulting evolution of the corona and inner heliosphere, to observe and characterize the Sun's polar regions and equatorial corona from high latitudes. The SO has the following in-situ instruments: energetic particle detector, magnetometer, radio and plasma waves measurement, solar wind plasma analyzer, extreme UV imager, multispectral coronograh, polarimetric and helioseismic imager, heliospheric imager, spectral imaging of the coronal environment, and StiX - X-ray spectrometer / telescope. The data of StiX are: mass $5 \mathrm{~kg}$, power $5 \mathrm{~W}$, dimensions $22 \times 22 \times 80 \mathrm{~cm}$, energy range $4-150 \mathrm{keV}$ (pixelized CdTe detectors), energy resolution $1-15 \mathrm{keV}$, field of view $2^{\circ}$, finest angular resolution 7 arc seconds, spatial resolution up to $1400 \mathrm{~km}$ on the Sun surface, time resolution $>100 \mathrm{~ms}$. STIX subsystems contain: X-ray window, imager, aspect system, attenuator, detectors and ASICs, instrument data processing unit, power supply, and full system simulator.
URSA OBC - an onboard computer for satellites: Further developments of a versatile self-reconfigurable digital processing platform for aerospace applications were summarized. Current trends for cheap satellite equipment are: increasing demand for computing power, fast communication channel internal and external including RF and optical, utilization for COTS components in LEO satellites, change of system functionality during the flight, needed re-configurability, usage of radiation immune components like ASICS and SRAM FPGAs, wide usage of popular interfaces to decrease the design effort and costs. Yet, the COTS components are radiation verified. The fundamental requirement of the system is the highest possible availability. Components of this fundamental requirement are: reliability, dependability, radiation endurance, multiple switchable functionalities, availability of own housekeeping and state monitoring, etc. A construction work is going on a universal pre-flight prototype called URSA. URSA uses antifuse FPGA and JTAG reconfiguration. Has an extended diagnostics and self-diagnostics capabilities including tracing of early wrong process and failure syndromes. It reports malfunction, performance and functional state to break down analysis block. In a case of need the decision block acquires new configuration changing or not changing the system functionality. The SRAM and RAM memories are under constant verification. Leon3 processor was used to run user software. It checks the functionalities of the Central Module of the Spacecraft, which is responsible for telemetry. URSA, which is a reconfigurable high speed digital processing platform with standard interfaces, in its pre-flight configuration, undergoes standard space-grade electrical tests, functional tests for all available FPGA configurations, thermal-vacuum chamber tests and vibration tests. The system passed typical acceptance tests for satellite equipment. Now it is subject to system level radiation tests including SEU, SEL and TID. The possible improvements include usage of larger antifuse FPGA and daughter boards.

Reliable AI based Power supplies for satellites: Onboard a satellite there is a need for several voltage levels. Different voltage values are provided by space-grade DC-DC converters, which are electronic circuits which convert a source of DC from one voltage level to another. It belongs to a class of power converters. There are linear and switching converters. The DC source on a satellite are batteries and solar panels. The following voltage levels are needed onboard a satellite: $7 \mathrm{~V}$ for a heater, $5 \mathrm{~V}$ for $\mathrm{X}$-ray sensor, $24 \mathrm{~V}$ for antenna, 3.3V for electronics, $12 \mathrm{~V}$ for a gyro. Satellite technology uses digital DC/DC converters, which have some advantages over analog ones: more reliable and stable in changing temperatures, less sensitive to aging and components degradation, increased resistance against radiation by using EDAC algorithms, smaller size, reduced mass, potentially smaller power consumption, possibility to use adaptive algorithms and optimization techniques. Digital DC/DC adaptability can: reduce the problem with phase lag leading to low phase margins, allow switching the controller from DCM to CCM and vice versa, optimize the converter by updating controller parameters depending on operating conditions, increase the system resistance to degradation and damage. Digital DC/DC converter consists 
of analog part - filters, drivers and switches and digital part - ADCs, compensator, and DPWM. Digital converter is a modular device and was implemented for a constant $50 \%$ duty cycle output. Cross line detector estimates the moment when the current slope crosses the calculated control value for current mode implementation. DCM/CCM crossing detection unit changes PID parameters when converter enters or leaves the DCM or CCM. GUI unit serves as an user interface allowing the user to set the controller parameters, output voltage level and ADC word length. OV unit detects overvoltage condition and switches the converter off. Reset switches on and off and resets other modules.

AI module synchronizes other units by sending requests to them in the right order. ADC unit is an interface for ADC converters. Filter module unit provides a simple digital filtering of the input data. Reference module calculates the error signal that is a difference between requested output voltage and its actual value. STD PID is a digital implementation of a PID controller with added high frequency pole. DWPM unit is a digital implementation of PWM with 5ns resolution. Digital DC/DC converters have two main drawbacks: increased jitter because of ADC noise and phase lag due to time delays between making a measurement of the output voltage and application of control effort in the power control stage. The delay results in the open loop frequency response of the converter as a rapid phase decrease for higher frequencies (phase roll-off). The converter bandwidth has to be limited by decreasing the crossover frequency, in order to keep phase margin at a desired margin. The limited bandwidth of the converter results in its larger transit response. Digital DC/DC converters suffer some problems while CCM/DCM border crossing. DCM converters have lower gain than CCM ones. DCMs exhibit lower efficiency under higher loads. The phase margin is increased by flexible voltage sampling. Implementation of DCM/CCM border crossing detection and automatic change of controller parameters (bandwidth increase) results in a possibility to change the frequency response of the device after it enters into different work mode. The device is equipped in the features: detection of degradation/failure of the output power stage, automatic switching from the $\mathrm{I}$ to the $\mathrm{V}$ work modes, overvoltage protection by applying additional crowbar circuitry for fast dumping the energy gathered in the power stage.

Reconfigurability of the onboard satellite equipment: One of the key factors in space applications of photonic and electronic circuits is the ability of dynamic partial FPGA reconfiguration. During such configuration it is to define reconfigurable partition boundary, fit-check all modules and generate. Proxy logic is done automatically while decoupling logic is done by the user. The outcome of this process is a set of bit streams: static, modules, full and black boxes. Process is started by the need for new functionality and freeing some resources to cover partial reconfiguration. Process is also started by detecting a malfunction, and isolation of the faulty module - fault mitigation. The function is moved to a new place in the matrix. A very good area for testing of partial reconfiguration are vendor DevBoards. The reconfigurability test setup consists of test monitor, input and output ports located on a PCB and source, sink and at least two areas in FPGA serving as reconfigurable partitions. The functionality can be switched back and forth. The question remains open if one can efficiently mitigate faults (radiation induced) in FPGA fabric by fast (blind or smart) scrubbing.

Off the shelf equipment: Astronomical equipment must fulfill the most stringent and highly normalized reliability standards. This increases dramatically the cost. On the other hand there is a tendency to use off-the-shelf high quality equipment. The currents trends are: high demand for computing power, utilization of COTS components especially for LEO (low Earth orbit) satellites, reconfigurability of the systems where the functionality depends on mission phase; usage of SRAM FPGAs for versatility and ASICs for reliability, usage of standard interfaces like $28 \mathrm{~V}$ bus, SpaceWire, CAN etc. The cost can be further decreased by applying universal systems. The requirements for such systems are: high availability and dependability versus unlimited reliability understood by large fault tolerance, switchable functionality, own housekeeping and state monitoring, usage of SpaceWire interface, availability of an integrated processor for running user software. The features of universal system for low price space applications are: reconfiguration of SRAM FPGA by antifuse (FLASH) FPGA using JTAG; verification of processing results and detecting syndromes; communication with other S/C modules. Communication tasks include: malfunction reports, acquiring new configuration, reporting of performance and functional state and executing superior module commands. The developed space oriented universal system provides basic functionality of all service modules at the spacecraft and it is its central unit for telemetry. It runs constant verification procedures of SRAM FPGAs configuration memory and onboard RAM memory. A pre-flight prototype of an universal system for space applications was manufactured at CBK PAN laboratory in Warsaw. There was verified re-programmability of memories in ProASIC FLASH FPGA. Re-programmability was checked to be less than $0.5 \mathrm{~s}$. There were verified several functions of the system: performance for on-board SEL effects protection, storage of FPGA configuration, clock source switching circuit and internal watchdog. The system undergoes radiation tests and integration in heat dissipating and radiation shielding package.

Image sensor tester for satellite telescopes: An universal image sensor tester was built as a platform for laboratory purposes for CCD matrices characterization. The purpose is to enable setting of proper operating points for matrix pixels, searching for bad pixels, lines, characterize distribution of sensitivities. Such a low cost tester is an invaluable tool for compensation of CCD characteristics, when the CCD matrix is used for measurement purposes like making spectral characteristics of unknown sources, also for astronomical applications. The key components of the CCD tester are: a wide range of efficient linear regulators, high speed CMOS drivers for charging/discharging the CCD capacitances, four-state high-voltage digital switches of three voltage levels and high impedance, digital I/O compatible with $5 \mathrm{~V}$ and 3.3V CMOS work voltage levels, SPI and $\mathrm{I} 2 \mathrm{C}$ buses, precision thermal controller made of Peltier modules with temperature stabilization, fast CDS 
and S\&H video ADCs, LVDS interfaces, camera link, fast and efficient data converter implemented in FPGA, dedicated GUI at a PC.

Astronomical databases: Astronomical databases get larger and larger with fast data accumulation form massive all sky observations. Satellite and ground based projects include deep space and wide angle sky monitoring. Specialized databases include measurement results for changing stars, results of GRB observations, quasars, standard candles monitoring, etc. A team from ISE applied CUDA (compute unified device architecture by Nvidia) for aiding the search in massive databases for weak spectral lines quasars (WLQ). Quasars, very distant objects, are fast rotating black holes surrounded by accretion disk, obscuring torus, emitting a strong jet along the rotation axis, with narrow and broad line emission and absorption regions - close to the accretion disk and jet area. Astrophysical objects change slowly, which enables unification theories based on statistical approach. Different features in quasar spectra, including the continuum and narrow spectral lines, are combined with various physical effects of large scale going on in the vicinity of the central black hole. Shape of the continuum reflects the processes in the accretion disk around the black hole. It is possible, from the spectra measurements, to determine black hole mass, spinning rate, singleness or binary set, matter inflowing rate, and observation angle against the spinning axis. The spectral emission and absorption lines are combined with the optical atomic and particle processes in the gas surrounding black hole. The spectra reveal the evolution of the objects as we observe them at different stages of their age. One of the questions to be answered here, by researching the WLQ spectra, is how faint non-active distant galaxy turns on its activity and becomes a bright quasar. The measurements determine gas density, constituents, emitters and absorbers, type of collisions, abundance, speed and direction of gas winds. The quasar spectral function has: continuum base $\mathrm{CB}$, narrow and broad emitting lines NEL, BEL, and narrow and broad absorption lines - NAL, BAL. All these shapes, lines and their characteristics are databased. WLQ are objects of typical continuum emission but with very weak or no features on the CB. They are considered as young objects without the jet. Typical databases for quasars have hundreds GB of data and are difficult for direct and efficient analysis. There is a need to process and compare millions of quasar like spectra of astronomical objects. For this purpose an Hbase was applied with unlimited column number. The data was adapted from changed PostreSQL. The numerable column tables, numbered in thousands, are backups of the dataframes in the $\mathrm{R}$ environment. The data is processed using its own matrix and multicore rules, good packages and with double precision accuracy. This procedure reduces the databases by a factor from 10 to 100 . Now a well known paradigm of using GPGPU technology was applied. Instead of using a CPU a GPU (graphical processing unit) was used for performing parallel computations on the spectral characteristics. In this approach, a one thousand column matrix containing one thousand spectra becomes a one-dimensional vector stream. This stream is loaded to the GPU. Kernel functions are applied to each element in the stream in parallel. The streams can be concatenated together, which results in shorter computation time. There are obtained linear indexes of column - major matrix with spectral data. The applied CUDA and Thrust library operates on high level streams and kernels. In the result 100 thousand space objects spectra were calculated 100 times faster, than using traditional in-series computational methods.

\section{Pi of the Sky Astronomical Telescope Network}

$\mathrm{Pi}$ of the Sky is a network of robotic optical telescopes. The webpage of the project is: http://grb.fuw.edu.pl. The telescopes and the project is run by a collaboration consisting of FUW, Warsaw University Astronomical Observatory, NCBJ, CAMK and WUT. All telescopes operate fully autonomously, without human intervention. Currently there are two telescopes. Working prototype apparatus is in San Pedro de Atacama SPDA in Chile. It was moved from ESO LCO ( European South Observatory, Las Campanas Observatory) in Chile where it worked for several years. The first telescope of the final system is located in INTA El Arenosillo test centre in Mazagon near Huelva Spain. Towards the end of 2012 there will be mounted three new units in the INTA location. The third observation site will be located near Malaga. Pi of the Sky research program embraces the following observations, measurements and cataloguing of: changing stars, transient astronomical processes, gamma ray bursts GRBs, gravitational waves detection, optical counterparts to other astronomical phenomena, cosmic rubbish, spectral analysis of bursting sources, photometric analyses of measured astronomical data, using parallax for measurements.

Photometric analysis of Pi of the Sky data: Pi of the Sky has gathered a lot of measurement data from optical observations at two locations in Chile LCO and SPDA and two locations in Spain INTA and Malaga. The stream of astronomical data acquired by $\mathrm{Pi}$ of the Sky telescopes is too big to be fully processed. It has to be reduced on-line and off-line, prior storing them for further analysis. On-line data reduction includes: flash recognition in real time analysis frame by frame; dark frame subtraction; fast photometry including numerical filter; comparison with reference image taken from series of previous images. Off-line data reduction includes: algorithms optimized for data reduction; adding 20 subsequent frames; dark frame subtraction; multiple aperture photometry (ASAS), astrometry, normalization to $\mathrm{V}$ magnitudes from TYCHO catalog; cataloguing of raw data to the database. Additional analysis is done by multilevel selection system to reject strong background like - fluctuations, hot pixels, cosmic ray hits, satellites. Photometry accuracy is significantly improved after removing bad quality data. Color correction and calibration algorithm is added to image improvement procedures. The assumptions are: no filters were used in cameras, detector response is correlated with the star spectral type B-V or J$\mathrm{K}$, catalog stars measurements are corrected for spectral type (R. Opiela, CFT PAS). To improve the data quality, a dedicated system of filters was built to mark bad measurements or frames. The data quality improvement procedure is applied to new data together with cataloguing procedure. Additionally the data quality is improved by applying approximate color 
calibration algorithm based on the spectral type of catalog stars. Statistical method was developed, taking into account all stars in the frame, allowing to reject bad quality exposures. The accuracy of photometry is increased to the level of $0.01-0.03$, by applying the new frame selection algorithm. A dedicated analysis of selected objects leads to further improvements of the measurement results. Quality of the data is good enough for research purposes.

Parallax in the Pi of the Sky project: Primary aim of the Pi of the Sky is to search for transient flashes in the sky of unknown origin and to determine the parameters of such events, like position, time, duration, distance, etc. Usage of the parallax effect is the most direct method to determine a distance from the observer to the object. There are no special assumptions to measure the distance. What is needed for measurements: directions of the lines of sight from two places, angle between them and distance between these places - called the base. The bigger the base the deeper one sees into space. Parallax method has a lot of main limitations. The parallax angle gets smaller with the distance very quickly. Parallax can be used only for relatively close objects. To improve parallax based observations, more powerful telescopes or larger base, or both are needed. Pi of the Sky North Observatory in Southern Spain in INTA location is in use since October 2012 (A. Majcher, NCBJ, Świerk). The new design of equatorial mount is carrying four cameras, designed by SRC, PAS. Two observational modes are used Wide and Deep. Pi of the Sky South observatory in Northern Chile at SPdA location (located $740 \mathrm{~km}$ North from LCO) is in use since March 2011. Since March $2011 \mathrm{Pi}$ of the Sky is able to observe a parallax of objects close o Earth. It allows these two observatories to reject false triggers, mostly originating from artificial satellites, rockets and space debris elements.

The base is huge, almost $8500 \mathrm{~km}$ along the Earth's chord. It is possible to observe parallax from $25 "$ to over $14^{\circ}$. Combination with camera diagonal results in an observable parallax angle for objects more than $20600 \mathrm{~km}$ from Earth centre. For closer objects, the parallax angle exceeds the image size. Combination with diagonal of the pixel results in an observable angle for objects over 38 million $\mathrm{km}$ from the Earth centre. For farther objects the parallax angle is smaller then the pixel size. Between these two limiting distances the system allows for observations of: geostationary and GPS satellites, space debris, the Moon, near Earth comets, planetoids, etc. The third Pi of the Sky observatory will be located in Southern Spain near Malaga, a distance around $249 \mathrm{~km}$ from INTA. Two Spanish Pi of the Sky observatories allow for observations of objects between 6800 and $870000 \mathrm{~km}$ from the Earth centre. LEO (low Earth orbit) objects, like ISS, HST and other, will be available for these observations using parallax. If observations were performed at the same time, i.e. fully synchronized, it is possible to determine a straight line connecting the observations with the object, which are simply parameterized. Then the position of the center of the vector connecting positions of their smallest distance can be determined by simple geometrical measurements. Due to huge parallax, the Pi of the Sky system observes precisely the parallax of geostationary satellites. Observations are done once or twice a night. Each observation lasts 90 minutes. Measurements are synchronized. The algorithm searches for flashes with maximal angular distance. Every flash candidate is verified in TLE NORAD satellites database. A list of candidates is published on the Pi of the Sky website. On the average, during 90 minutes of observations, Pi of the Sky telescopes record almost 3000 different satellites. It is possible to determine the distribution of difference between the satellite distances known from the TLE database and the distance $\mathrm{R}$ determined by the Pi of the Sky algorithm.

Pi of the Sky as a gravitational wave telescope: Pi-of the sky project of wide angle sky observations in the optical spectrum is used now for gravitational waves searches (A. Zadrozny, CAMK, PAN). Gravitational waves are still undetected. Some of detectable gravitational wave sources are expected to have EM counterpart. The pi-of-the-sky observations base on this assumption. Gravitational waves are assumed to come from binary coalescence and supernova explosions. Detection of gravitational waves base on large optical interferometers like LIGO. The method bases on locating and observing optical counterparts to un-modeled pulses. The involved experiments in the Looc-Up Project, for runs spanning from 2009 till now, are: TAROT North in France and Tarot South in Chile, QUEST - Chile, Swift, Skymapper, Pi of the Sky - Poland, ROTSE, PTF, ZADKO - Autralia, Liverpool, LOFAR and LUMIN. Lumin, for example is a system for very fast locating and positioning of an event on the sky with medium accuracy. The aim is to alert big telescopes for directed observations and for helping the operators to make decision about the observations. Now, Lumin sends up to one alert a day. The succession of work of Lumin is as follows: data generation and transfer, identify triggers, write to database, select triggers and determine positioning, send alerts to telescopes. The total latency is less than 30 minutes. The major contribution to the latency is by manual event validation. The models of the possible sources are: long GRB, long GRB off axis, short GRB, short GRB off-axis, supernovae, kilonovae, and other.

Pi of the Sky has four cameras with the FOV 20x20 deg, exposition time $10 \mathrm{~s}$, limiting brightness $12 \mathrm{mag}, \mathrm{CCD} 2 \mathrm{Kx} 2 \mathrm{~K}$, observation site is near Warsaw. Catalog based pipeline is used for analysis. Guide Star Catalog with stars up to 11 mag is used as a seed for star catalog. For each exposition all recognized stars are added to the database with their brightness measurements. Normalization of brightness to $\mathrm{V}$ magnitudes is from TYCHO catalog. Transient search concerns: new objects that fulfill quality constraints, objects that suddenly increase their brightness. All transients are inspected manually. Objects that are suspected to be transients are cross-correlated with INTA (Spain) images (4 cameras), taken later than the science runs. Due to wide field view, with pi-of-the-sky system it is possible to image huge part of the sky within less than an hour. On-line transient recognition might be essential in science runs for effective transient observation and recognition. The main aim of the project is to make externally triggered search connected with optical transients of unknown astronomical origin. Currently the data is available from the Pi-of-the-Sky telescope. The data includes: 40 optical transients observed from the Las Campanas Observatory in Chile, 31 transients 
observed from San Pedro di Atacama (Chile). Most of these transients have duration of less than $10 \mathrm{~s}$. The sources are offaxis short GRBs or orphaned after glows from such GRBs, or some unknown astronomical events. Two sites of pi of the sky telescopes located away of $100 \mathrm{~km}$ from each other in Spain allow for using the parallax for effective measurements. Piof-the Sky project is part of two gravitational wave search projects: Look-Up and Optical Transient ExtTrig Search. Published results from both projects are expected in 2013. Pi ofthe-Sky is the biggest FOV telescope that is taking part in gravitational waves search.

Pi of the Sky upgrade - Icinga: An advanced monitoring system is under construction for Pi-of-the-Sky experiment (A. Ćwiek). The monitoring system will supervise all hardware and software, i.e. any device or service for which one is able to write a plugin that reads the parameters and returns them. What is monitored: each telescope, status of the dome, status of computers that support the telescopes, mount status, camera status like chip temperature, frame size, number of stars, astrometry. The slow control parameters are too many to be controlled manually for each telescope. The control requirements are met by Icinga. Icinga is a fork of Nagios and is backward compatible. It is an enterprise grade open source monitoring for network and network resources. It notifies the user of errors and recoveries, generates performance data for reporting. It is scalable and extensible, can monitor complex, large environments across dispersed locations. It stores data in text files, MySQL, PostgreSQL, and Oracle. Icinga architecture consists of three components: Core, API, Web and common database IDOIDB (Icinga data out database). Icinga core manages monitoring tasks, receives check results from various plugins, communicates these results to the database. Icinga API fetches information from the core to the database and supports various interfaces from database PHP PDO output to pipe and SSH input. Icinga web is an online portal to view Icinga monitoring results and send commands to the core. Thanks to the loose bundling of Icinga core, web, API and database, these components can be distributed and connected by a switch or any other intermediary. Such systems ensure that monitoring system itself is fail safe, so should one component fail out, it can be replaced without disturbing the system as a whole. The applied Icinga system allows to continuously monitor vital parameters of detectors and computers which control them in $\mathrm{Pi}$ of the Sky experiment. Icinga facilitates fast response to anomalies or failures in experiment performance. Automatic recording of parameters of all components of the Pi of the Sky experiment allows an efficient identification of possible causes of failures.

Development of the next generation of pi-of-the-sky ultrasensitive cameras require building a photonic and electronic test stand for precise qualitative camera sub-system evaluation. One of the tasks here is to build temperature stabilization device in calibrated source using spectral method. Calibrated light sources tend to change their parameters during work. The reasons are: temperature changes during the work of the source and wearing out of the source. The source radiation spectrum shifts towards shorter wavelengths and the radiation intensity increases with higher temperatures An additional feedback control circuit was added with the aim to correct the light source work conditions. The device consists of an integrating sphere for intensity measurement and spectrum acquisition, two photodiodes and a spectrometer; two shutters (coarse and fine), driven by stepper motors, for intensity regulation; halogen lamp current control circuit, and DDC112 integrated circuit for photodiode current measurement. The driver, which uses SPI interface and bases on ATmega64@16MHz, several DACs and ADC, UART, digital I/Os, several sensors, LCD 4x20 matrix for characters display, handles the setting of correct work conditions for the light source. It is done by current control, shutter control, voltage monitoring and light intensity monitoring. The driver has duplex communication bus with the spectrometer LPC1764@100MHz. The spectrometer handles spectrum measurement, calculates optimal work conditions for the light source. The system prototype worked on a PC and then was transferred to ARM with MatLab algorithm.

Global Robotic Telescopes Array - GLORIA AND LUIZA: Gloria is an FP7 project Global Robotic Telescopes Intelligent Array for e-Science [Gloria-project.eu]. Luiza is data processing system for Gloria. The coordinator of Polish participants is prof. F. A. Żarnecki of UW, Faculty of Physics. It gathers 13 partners and 17 robotic telescopes in Europe, Africa, South America and New Zealand. The project goals are: create free and open-access network of robotic telescopes for citizen science, to develop Web2 environment for easy access to telescopes and other network resources, design tools for doing on-line experiments - observations, and off-line analysis, develop full framework for doing research with robotic telescopes allowing also for easy integration with Gloria network, outreach in science and astronomy. The approach is based on earlier Cyclope project, located at Montegancendo Astronomical Observatory, UPM, Madrid. Data processing concept in the system is based on HEP experiments, like LHC or ILC, which gather enormous amounts of data. Marlin framework is developed for efficient data reconstruction, reduction and analysis. The aim is only relevant event building out of huge amount of data. Merlin is based on the following approach: data reconstruction and analysis should be divided into small, well defined steps, implemented as so called processes; each step has to have well defined input and output data structure; by defining universal data structures one makes sure that different processors can be connected in a single chain analysis allowing for data exchange and analysis results; processors configuration and their parameters can be set by user at run time in a simple steering file. The same concept is used in Luiza framework dedicated for Gloria. Efficient and flexible analysis framework for Gloria was developed basing on HEP concept. Basic structure of tools were implemented, ready to work on Gloria experiments. First public version is available. First off-line experiments with Luiza are scheduled for September 2012.

Spectral characteristics of X-ray bursters: Spectral analysis allows astronomers to determine the mass and radius of a neutron star. In consequence, physics of super dense matter is revealed, as well as more precise models of neutron stars and binary stars can be created. The masses of neutron stars show strong concentration around 1.4 solar masses, at 
least for pulsars. Theoretically, the mass of a neutron star can be in the range of $0.1-3.0$ solar masses. $X$ ray busters were discovered in 1976 by Grindlay et al and Belian et al. These sources are neutron stars in interacting binaries. The companion star has low mass. The neutron star has a weak magnetic field. During decay of the burst the spectrum becomes softer, what is classified as type I of the burst. X-ray bursts are recurrent events but not strictly periodic. Time interval between the bursts are typically $10^{4}-10^{5} \mathrm{~s}$. Energy released per burst is around $10^{39} \mathrm{erg}$. The source of the Xray burst is a thermonuclear flash. The burst spectrum spans in the area of $0-20 \mathrm{keV}$ photons, with maximum from 5 to $7 \mathrm{keV}$. An example is MXB 1728-34 X-ray source discovered in 1976 during Uhuru sky monitoring. The data is: neutron star, atoll type, 4-8h time between bursts, spin frequency $364 \mathrm{~Hz}$, optical counterpart not observed, distance $4.2-5.1 \mathrm{kpc}$, no superbursts present. The assumptions for spectrum research were as follows (A. Majczyna, J. Madej, M. Należyty, NCNR, WUO). Analyzed spectra were integrated over $0.25 \mathrm{~s}$ at the end of bursts. The model simplifying assumption is that the atmosphere is static with constant temperature. Accretion disk and flow was neglected. Only the neutron star atmosphere is a source of photons. The neutron star is non rotating. Magnetic field does not modify opacity coefficients. Relativistic corrections are not included in model atmosphere. F-f, b-f and $b-b$ processes were included and Compton scattering. Photons are scattered on relativistic electrons with thermal velocity distribution. Large relative energy and momentum exchange between photon and electron is allowed during a single scattering event. Analysis of spectral characteristics with these assumptions allow for the determination of neutron star parameters, which are independent on the distance. The core radius can be determined without the radius of the emitting area. The best fit is obtained for the models with $\mathrm{He} / \mathrm{H}=0.11, \mathrm{Fe} / \mathrm{H}=10^{-3}$. One sigma confidence model gives approximately $\mathrm{M}=0.1--1.5 \mathrm{M}_{\mathrm{s}}, \mathrm{R}=2-10 \mathrm{~km}$. These parameters are in agreement with EOS of strange quark matter.

\section{Accelerator Technology And High EnERgy PHYSICS EXPERIMENTS}

High energy physics (HEP) experiments involve big infrastructure like superconducting accelerators and detectors. At least $10 \%$ of the costs of these infrastructures are consumed by electronic and photonic systems for the construction of detectors, control and measurement systems, reference timing and synchronization systems, safety and protection systems, etc. The biggest and most important research processes going on now in the domain of HEP are associated with the Higgs boson discovery and neutrino oscillations. Both fields, apart from some other ones, are expected to lead to the new physics and open new clues to explanations of such unknown fields as dark matter and dark energy. Also big research infrastructural investments concern free electron lasers. The biggest one in Europe is the E-XFEL under construction in DESY.

There are very strong motivations for the research on free electron lasers. Photons of different wavelengths are one of the most useful tools in different types of measurements. IV generation light sources are the only ones to generate coherent, very intense laser light from IR, visible, through $\mathrm{UV}$ up to $\mathrm{X}$ band. This is not achievable in any other type of laser. In FEL the light is generated by the energetic relativistic electrons traveling in the undulation path, during SASE process (spontaneous amplified self emission). The electrons are accelerated to relativistic energies in a linear accelerator.

\section{THE CMS EXPERIMENT}

Higgs search by the CMS experiment: CERN has just announced the discovery of the Higgs like boson with no spin, with the mass of $125.3 \pm 0.6 \mathrm{Gev} / \mathrm{c}^{2}$. This is very probably the first boson of the Higgs family. The announcement is based on combined data from the CMS and ATLAS experiments. Warsaw Group (UW, Faculty of Physics, NCBJ, WUT) is one of the founding members of CMS Collaboration. The CMS (compact muon solenoid) is running in parallel to ATLAS experiment, both of them located on the LHC ring accelerator (large hadron collider), both are optimized to hunt for the Higgs particle. The LHC potential of discovery in now unrivalled. Among the potential discoveries are: Higgs boson, heavy supersymmetric partners of elementary particles, weakly interacting charged heavy particles, non-barionic candidates for dark mater particles, dark photons, new properties of neutrinos, phase boundaries and properties of QGP (quark gluon plasma), micro dark holes, potential internal structure of quarks, and many more. Higgs particle is linked to the masses of all particles in the baseline SM (standard model). There are several channels to observe potentially Higgs particle production and decay. The dominant production mechanism is gluon fusion $\mathrm{gg} \rightarrow \mathrm{H}$. The best channels for Higgs searches are: $\mathrm{H} \rightarrow \gamma \gamma, \mathrm{H} \rightarrow \mathrm{WW} \rightarrow 212 \nu, \mathrm{H} \rightarrow \tau \tau, \mathrm{H} \rightarrow \mathrm{bb}$. The golden channel, for all Higgs masses, is $\mathrm{H} \rightarrow \mathrm{ZZ} \rightarrow 4 \mu$, or generally 41 $(4 \mathrm{e}, 2 \mathrm{e} 2 \mu, 4 \mu$ ). Basing on the 2010-2011 and part of 2012 publically available CMS data the existence of Higgs particle is excluded at the confidence level $95 \%$ in Higgs mass range $127-600 \mathrm{GeV}$. The excess of events is visible at masses around $124 \mathrm{GeV}$. Till now CMS has not noticed any deviations from the SM. Some results come from CMS HI (high intensity) program.

A team from Warsaw Universities (WU and WUT) is participating in the maintenance of the CMS experiment and development of some of its internal detectors. CMS inner detectors, situated inside a superconducting coil with the field of 4T, are: Tracker - silicon microstrip pixels, ECAL scintillating $\mathrm{PbWO}_{4}$ crystals, HCAL - plastic scintillator brass sandwich. The detectors outside the coil, situated in the field of 2T of opposite sign are: muon barrel and muon endcaps - interleaved with iron yoke. They consist of detector layers: DT - drift tube chambers, CSC - cathode strip chambers, and RPC - resistive plate chambers. Particles are searched with big transverse momentum in reference to the bunch crossing longitudinal axis. Different particles deposit their transverse energies in different layered detectors adjacent and more distant from the interaction point. Silicon tracker determines the individual vertex of each interaction with some accuracy in 
space and time. Then the particles reveal their passage through CMS. Photons and electrons are trapped in the Electromagnetic Calorimeter (ECAL). Neutrons deposit their energies in the Hadron Calorimeter (HCAL). The most permeable particles of these, the muons leave their tracks behind in the Muon Chambers. Outside the superconducting solenoid the iron return yoke is interspersed with muon chambers. DT response time is $380 \mathrm{~ns}$ and spatial resolution $100-150 \mu \mathrm{m}$. RPC response time is $2 \mathrm{~ns}$ with effectiveness $>95 \%$. CSC response time is $3 \mathrm{~ns}$ and resolution $150 \mu \mathrm{m}$. The RPC are fast gas detectors for efficient muon measurements. Their layered structure consists of isolator, graphite, Bakelite, gas chamber, readout strips (width $0.5-4 \mathrm{~cm}$, length $20-130 \mathrm{~cm}$ ) and high voltage $\mathrm{Al}$ foil. The supply voltage is $9.5 \mathrm{kV}$. Noise is smaller than $5 \mathrm{~Hz} / \mathrm{cm}^{2}$. Muon tracks are recorded in the CMS. Inside the solenoid the magnetic flux density of 4T bends tracks of charged particles. In the outer region the magnetic field is directed the opposite way, which causes a bend in the other direction. The bending radius is a function of charge to mass ratio. Muons with transverse momentum $\mathrm{p}_{\mathrm{t}}<3.5 \mathrm{GeV}$ are bent inside the solenoid. Muons of the transverse momentum in the range of $4<\mathrm{p}_{\mathrm{t}}<8 \mathrm{GeV}$ are following less and less curved path. Muons are fast (relativistic) and leave tracks inside their bunch crossing window. The key sub-system for particle detection in CMS is TRIDAQ (trigger and data acquisition). Coarse data from particle collisions go to the FLT. FLT (first level trigger) is dedicated electronics consisting of ASICs and FPGAs, working at $40 \mathrm{MHz}$, and doing only logic functions. FLT analyses every event taking place at each bunch crossing BX. The processing is pipeline type with latency $3 \mu \mathrm{s}$, including $2 \mu$ s for optical fiber data transmission between the detector and counting room. The output from FLT is $100 \mathrm{kHz}$. The data are stored in readout buffers for 128 events. DAQ readouts the data for the selected events (the events are fragmented). Event Builder is a switching network which gathers data from one event into one HLT computer. HLT (high level trigger) is a huge computer farm, consisting of more than $10^{3}$ nodes, $10^{4}$ cores and nearly 20TB memory, which runs the software events selection algorithms. The output from HLT, substantially smaller than $1 \mathrm{kHz}$, is stored on a permanent memory. FLT is made of custom electronics and is pipeline at $40 \mathrm{MHz}$, and multilayer including calorimeter trigger, muon trigger and global trigger. The role of trigger system is to identify, measure and sort the trigger objects. Global trigger applies cuts for single or multiple objects and searches topological correlations.

CMS RPC Muon Trigger: Warsaw group works on RPC PAC muon trigger. The signals from FEB (front end boards) connected directly to RPCs detectors go to Link Boards via LVDS cables. Then, $10^{3}$ optical fiber cables carry the $1.6 \mathrm{Gbps}$ signals to the counting room, where there are 84 Trigger Boards located in 12 Trigger Crates. Trigger algorithm bases on pattern comparison in pattern comparators PAC. The chamber signals (fired strips) are compared with the predefined set of patterns. Each pattern has assigned a transverse momentum pt and sign - depending on the track bending by the magnetic field. A pattern is a set of AND gates connected to selected strips. Muon candidate is recognized if RPC hits fit to the pattern and are in the same $25 \mathrm{~ns}$ clock period (BX). A candidate is produced even if not all layers have hits. The minimum required number of fired layers is 3 (out of $3,4,5$ or 6 layers available - depending on a detector region). In this way the trigger efficiency does not suffer from the limited geometrical acceptance and inefficiency of the chambers. The number of fired planes defines the candidate quality. The quality is used for the candidates sorting and "ghost busting" - which is cancellation of duplicated candidates. The trigger algorithm is implemented in the FPGA circuits. 300 chips are needed to cover full detector. Each PAC chip comprises up to 576 chamber strips and contains $3-14 \times 10^{3}$ patterns. Out of these patterns most concern low $\mathrm{p}_{\mathrm{T}}$. The patterns are stored in the firmware logic. Each PAC contains different patterns, therefore a separate compilation is needed for each chip. The patterns are generated basing on the simulated muon track. Advanced algorithms are used to create the patterns from the simulated patterns hits, assign $\mathrm{p}_{\mathrm{T}}$, and then select optimal set of patterns. The goal is to achieve best possible trigger efficiency and purity with patterns set that can be fit into the PAC FPGAs. The software framework for patterns generation and firmware compilation on the computer cluster was created. As the PAC algorithm is implemented in the reprogrammable FPGAs, it can be easily changed, e.g. corrected, improve performance, implement new features.

The system has to deal with SEU mitigation problem. Link boards and control boards are exposed to radiation and therefore can be affected by SEUs. Experiments show that configuration memory of FPGA chips are susceptible, while flash memories are unaffected. To mitigate the effects of SEUs we have several measures in place: triple modular redundancy in critical paths; re-reading of configuration registers in the background and check for flipped bits; checksum of all data stored in flash; periodic reloading of configuration from flash memories in a synchronized way and detector-wide. To make reconfiguration faster and independent from the control software running on PCs we have small automaton, which can read commands from flash memory and set values of registers, communicate over I2C and so on. Reset signals are transmitted together with the clock signal over TTC (fiber optic) and do not depend on the bus used for usual communication with PC software (CCU).

The trigger system has to be fully and precisely synchronized. The time of muon flight from the point to the different chambers varies from 14 to 42 ns, i.e. more than a single BX. This stems from the dimensions (muon travel path) varying from 4 to $14 \mathrm{~m}$. The time of signal propagation from the chambers to the Link Boards varies from 33 to $107 \mathrm{~ns}$, due to the difference in cable lengths. The chamber hits must be in the coincidence on the PAC input to produce the muon candidate. They have to be recognized as originating from the same BX, which means $25 \mathrm{~ns}$ clock period. Thus, the system synchronization is crucial for triggering performance. The chamber hits are synchronized to the $40 \mathrm{MHz}$ LHC clock in the link boards. The hits are quantized to the full BX (the timing is measured with the $25 \mathrm{~ns}$ precision) with the use of synchronization window. Window position can be adjusted with $0.1 \mathrm{~ns}$ accuracy. The hits are then aligned between the 
Link Boards (LBs) by applying full BX delays. The goal is to have all hits of all muons from given event within $25 \mathrm{~ns}$ on all LBs. The initial position of the synchronization window is calculated from muon hits timing (muon time of flight and signal propagation in cables), known length of optical fiber transmitting the clock. After the initial synchronization in most of the LBs the chamber hits are concentrated in one or two neighboring BXs: $-1,0,+1$. The timing correction is calculated by comparing the hits distribution from data with distributions obtained from timing simulations. In practice, LB synchronization is tuned very rarely and the residual out-oftime hits originate from cosmics, muons from other BXs or chamber noise. The upper limit for the pre-triggering and posttriggering is $0.04 \%$. The efficiency of RPC PAC trigger for identifying muons is a convolution of: geometrical acceptance of the RPC detector (fraction of muons crossing at least 3 chambers), chamber intrinsic efficiency, patterns efficiency probability that the chamber hits of a triggerable muon fit to any pattern. RPC total efficiency is $92 \%$ in barrel and $80 \%$ in endcaps. A triggerable muon is identified when hits are in at least 3 RPC layers inside the eta-phi cone covered by one PAC unit and appearing in the same BX. The current performance (as of 2011) of the RPC PAC trigger is very good. The work is continued on the pattern optimization with increasing LHC luminosity and higher muon rate and keeping high trigger efficiency for high momentum muons.

Search for Heavy Stable Charged Particles: Another modification of the PAC algorithm is motivated by search of new particles (A. Zagoździńska, ISE PW). Some supersymmetry models foresee Heavy Stable Charged Particles (HSCPs), like stop, gluino, stau. Their mass is of the order of hundreds $\mathrm{GeV}$, thus if they are produced at the LHC their velocity would be significantly smaller than c. In the CMS these particles will look like slow muons. Their hits in muon chambers (all or only outermost) can be up to $1 \mathrm{BX}(25 \mathrm{~ns})$ later than the hits of the regular muons. Thus, they will not produce the muon trigger, because the hits are not in coincidence within one BX. The alternative is that they will produce muon trigger but $1 \mathrm{BX}$ later. Thus, the tracker hits will not be recorded, because pixel detector stores hits from a single BX event only. There is a new algorithm to trigger on the HCSPs with the PAC trigger. In the PAC logic, the detector signals are extended in time to 2BXs. On the Global Muon Trigger input the PAC candidates delay is reduced by $1 \mathrm{BX}$ with respect to the DT and CSC candidates. Hits of the late particle generate trigger in the proper BX. Intime muons candidates appear in two BXs. The first candidate is too early and the second is in the proper BX. The first candidate is masked on the GT by the BPTX veto - a signal synchronous with collision, but advanced of 1BX (used for all triggers to eliminate the pre-triggering). Significant increase of the efficiency is obtained to trigger on lower momentum, slower moving super-symmetric particles.

\section{TOTEM EXPERIMENT}

TOTEM experiment - upgrade of data acquisition system. Upgrade of the Data Acquisition System of the TOTEM experiment includes the Scalable Readout System (A. Fiergolski.
CERN and ISE WUT). The signals are carried via GbE from detector to the S-Link Mezzanine OptoRx bords. These are connected via PCIe-PMC LVDS connector to FEC, and further via DTS $800 \mathrm{Mbps}$ to SRU, Second Level Trigger, and next to a PC and disk array via GbE 4x5Gbps. Opto-Fec card was designed. The test board links the OptoRx and the FEC and features the following components: 8xLVDS channels, fullduplex 2Gbps SERDES, clock generator and jitter cleaner, 64-bit parallel bus, TTC interface, I2C configuration, TTS support, JTAG support and independent power supply mode. There is a proposal of the new OptoRx firmware which would include second level filtering algorithms and new, more efficient data formats. The new OptoRx architecture would include: easily extendable output interfaces, AXI, and would base on the experience with TOTEM detectors. Testing environment (testbench) was designed to research new releases of the OptoRx firmware. The emulation environment of the detectors uses: SystemVerilog IEEE 1800, UVM - Universal Verification Methodology - Accelera, and TLM - transaction Level Modeling.

\section{ATLAS EXPERIMENT}

ATLAS TRT - transition radiation tracker: Detector control system for the ATLAS transition radiation tracker was designed and implemented by the TRT DCS Team (E. Banaś, Institute of Nuclear Physics PAS, Kraków). TRT is the outermost of the ATLAS inner Detector Tracker. It is a gaseous detector providing tracking through individual drift tubes (straws) and particle identification via detection of transition radiation. It is positioned in barrel, end cap A and end cap C of ATLAS and contain nearly 300000 straws. Each straw, filled with active gas (Xe, $\mathrm{CO}_{2}, \mathrm{O}_{2}$ ), is a proportional counter with the wall biased to over $1.5 \mathrm{kV}$ and wire grounded. Signals from the straws are read with the TRT front end electronics, nearly 900 FEBs. The DCS consists of many subsystems as follows: gas gain stabilization system, finite state machine, HV and LV software interlocks, TRT temperature hardware interlock, etc. The TRT DSC is operating the detector in a very stable way and reliably. The system is subject to continuous growing and developing, like new tools are added, together with the detector needs. System upgrade is predicted for long shutdown of the LHC during 2013-14. New front end hardware is predicted for this upgrade as well as new communication software (OPC UA) and move from XP to Linux, where available.

ATLAS TriDAQ: Discrete event modeling method was used for ATLAS TDAQ architectures design (K. Korcyl, Kraków University of Technology and INP, PAS). DEM is a software system simulating system dynamics in time, where state of the system remains constant between events and the processing system in a state may lead to scheduling a new event in the future. ATLAS TriDAQ is now exploited successfully in the LHC system. The system consists of FE pipelines, readout drivers, readout links, readout buffers and sub-systems, dataflow manager, event building network, sub-farm input, event builder (EB) and filter, sub-farm output and data storage. The first level trigger works at LHC frequency of $40 \mathrm{MHz}$. HLT reduces the frequency subsequently to $75 \mathrm{kHz}, 2 \mathrm{kHz}$ and 
200Hz. The HLT (high level trigger) includes RoI builder, L2 supervisor, network and processing unit, and finally event filter processors. 500 CPUs are used for LVL2 and 100 CPUs for the EB. Alternative architectures for the LVL2 network are switch or bus based. The researched issues are respectively: scaling, reliability, number and types of switches for the first case, and network traffic granularity and potential congestions for the second case. A test bed was prepared for checking the options including measurements of EB rates (from 1 to $6 \mathrm{kHz}$ ) for various accept ration by the triggers. Full scale switch based model was simulated and measured. Discrete event modeling becomes invaluable tool in design of large scale TriDAQ systems for modern high energy physics experiments. Modeling of ATLAS LVL2/EB traffic network helped to design and implement efficient network, which was recently checked by real new physics discovery of Higgs like particle.

\section{PANDA EXPERIMENT IN HESR FAIR}

Event Builder in PANDA - FAIR: The Research Group from Kraków (K. Korcyl) participates in building of PANDA detectors. This is a particle identification experiment at HESR - High Energy Storage Ring in FAIR - Facility for Antiproton and Ion Research at GSI Darmstadt. The particle identification is based on: DIRC - detection of internally reflected Cherenkov, ToF - time of flight system, MD - muon detection system, RIC - ring imaging Cherenkov Detector. The tracking detectors are: Micro Vertex Detector, Central Tracker, GEM stations (gas electron multipliers), Forward Tracker. The trackers are supplemented by the Electromagnetic Calorimeter. The PANDA DAQ requirements are as follows: interaction rate $20 \mathrm{MHz}$ at the luminosity $2 * 10^{32} \mathrm{~cm}^{-2} \mathrm{~s}^{-1}$; typical event size $4 \mathrm{kB}$; expected throughput around $100 \mathrm{~GB} / \mathrm{s}$; high flexibility in event selection for rich physics program; front end electronics and photonics working in continuous sampling mode; and lack of hardware trigger signal. The architecture is of push only type and consists of: detector FEE, data concentrators, FEE stage, CPU stage, event processing farm. The system is served by SODA - photonic time distribution system. It is a passive point-to-multipoint bidirectional fiber network providing time reference with precision better than 20ps and performing synchronization of the data taking process. The hardware of choice is ATCA standard (advanced telecommunications computing architecture) with full mesh backplane. Compute Node board in equipped with 5 FPGAs and provides high bandwidth connectivity by multi gigabit optical links connected to RocketIO ports and 5 GbEth links. Inter-crate wiring is designed in such a way that the module in slot $\mathrm{N}$ at the FEE level connects, with two links trunks, to module at slots $\mathrm{N}$ at the CPU level. The odd event packets at the FEEE level are first routed via the backplane and then outbound to the CPU level via a proper trunk line. The even events packets go outbound to the CPU level first and then use backplane to change the slot. The ports are fully parameterized adding flexibility to the design. Models of data source (data concentrator) and data sink (event building CPU) were designed and realized for system work simulations. Work parameters were estimated like: event building latency (architecture is able to switch up to $173 \mathrm{~GB} / \mathrm{s}$ ), load distribution between CPUs, monitoring queues evolution (averaged maximal queue length in input ports at the FEE level), monitoring of optical fibers link load, and monitoring FPGA links occupation. In PANDA project the Group from Kraków used modeling to demonstrate that the push-only architecture with Compute Node in ATCA offers over $100 \mathrm{~GB} / \mathrm{s}$ throughput needed to perform burst and super burst building and to run selection algorithms on fully assembled data.

\section{Neutrino Oscillation Research}

Warsaw Group (IRE WUT, prof. K. Zaremba, prof. J. Marzec) takes part in the neutrino T2K experiment. The goal is to find the components of the neutrino mixing matrix. The neutrino beam is propagated from J-Parc to Super Kamiokande for $295 \mathrm{~km}$. The Group participated in the construction of the T2K Near Detector - ND280. The detector, situated $280 \mathrm{~m}$ from the source, consists of: one gas detector and five fiber optic scintillating detectors. The fibers are surrounded by volume scintillators and coupled to MPPC detectors.

\section{LHC ACCElERATOR SyStem Development}

Development of Reliable LHC Accelerator Protection System Photonic and electronic systems are applied in protection systems for high energy particle accelerators. The key role are playing photonic active components and PLDs. Safe Machine Parameters System (SMP) is crucial for the LHC (large hadron collider) operation. It protects the LHC during injection procedure and during normal operation. It is modular, redundant VME system with two independent FPGA chips on each VME board - with monitoring and control tasks. The system receiver redundant information, generates flags and values. It is distributed around the LHC to be used by other protection equipment.

During the SMP development the safety approach was required (Protection System Lifecycle) which included: PSL based on the standard IEC-61508 Lifecycle, good understanding of the EUC, identify hazards, determine protection functions (PF) specifications. The requirements of the Protection Functions are requested by the Machine Protection Panel - which consists of physicists, engineers, risk analysts and decision makers. The focus is on the PF implementation in the PLD devices and systematic failures avoidance. The formal design flow includes: definition of requirements, assessment of critical and non-critical safety factors, preparing specifications, formalization of the process, building functional blocks of the system. All these steps undergo thorough check for completeness and consistency. Then, the process goes on via blocks implementation and simulation. Hardware stages include blocks hardware testing. The full system is then simulated, hardware is again tested in full and the code is reviewed. The whole system simulation and hardware tests process is externally checked for correctness. Thus, there are two levels of testing: in a simulation test bench which is a software wrapped around the model, and in a hardware test bench, which is similar 
to simulation but concerns the real hardware. The system response at the simulation and at hardware levels should be correct for all stimuli. The simulation tool can examine code coverage. The hardware tool uses embedded logic analysers provided by FPGA vendors (ChipScope, SignalTap, etc). In summary, the approach to dependable PLD design goes on top of dependable electronics design and consists of the following levels: formalization of the specification, splitting of risk factors to critical and non-critical, reduction of the problems to minimum functions, exhaustive source code simulation, full code coverage, hardware testers, code reviews, external reviews.

\section{WhITE RABBIT}

White Rabbit is expected to be an enabler of a number of innovative technologies and applications in photonics and electronics. WR uses two protocols for synchronization: synchronous Ethernet and enhanced PTP. The clock is encoded in the Ethernet carrier and recovered by the PLL of the PHY, which means no extra traffic cost. There are offsets between the clocks to be managed. PTP synchronizes the local clocks with the master clock by measuring and compensating the delays introduced by the network links. One of innovative WR based solutions is a precise local positioning system based on White Rabbit. WR is a deterministic Ethernet-based network, which synchronizes up to 1000 nodes with subns accuracy over fiber or/and copper, to a distance up to $10 \mathrm{~km}$. The application consists of a WR switch, a network of distributed receivers, a transmitter and an embedded computer. Low power transmitter generates a pseudo-random signal. The receiver detects the signal and transmits the information to the computer. WR switch synchronizes the receivers and performs further data transfer. The embedded computer receives the data from the SPEC cards and estimates the position of the transmitter. The receiver uses an FPGA processor. The FPGA controls SDR module, correlates the received signal with the pattern, does the time-stamping of the signal and transmits the packets. The following components and software pieces were used for construction of the receiver: simple PCIexpress FMC carrier with PPC (power PC CPU) - SPEC, FMC slot, XS6 FPGA, SFP transceiver with WR support, FMC card with SDR (software defined radio), SDR implementation and SPEC firmware. SPEC board is an endpoint of WR network. SPEC PCB contains: FPGA, SFP connector for WR network, FMC connector for external interfaces and modules, PCIe connector. SPEC is a PCIe device and needs a PC platform to work. Our SPEC design was extended by putting there a PPC CPU to make it a standalone system. Thus, the FMC carrier with PPC CPU is an independent embedded system that can work as an endpoint of the WR network. It may be also used as a measuring system and can be extended through the FMC connector. The system was tested in real life working conditions. The system supports also non-WR devices, i.e. it is compatible with the Best Effort non-synchronous Internet as well as synchronous Internet (high quality and low latency for high performance computing).

$\mathrm{WR}$ is a synchronous and deterministic version of the Ethernet. It is back compatible with the classical Ethernet.
White Rabbit is predicted for accelerator control and timing. It is developed by international collaboration (M. Lipiński, CERN and ISE WUT), based on well-known technologies and on open hardware and software. The main features are: transparent, high accuracy synchronization, low-latency, deterministic data delivery, designed for high reliability, and plug and play. The existing and near future as well as potential applications of WR installations are: CERN neutrino to Gran Sasso, GSI, HiSCORE - Gamma and Cosmic Ray Experiment in Tunka, Large High Altitude Air Shower Observatory in Tibet, Cherenkov Telescope Array, ITER, European Deep Sea Research Infrastructure - KM3NET. The WR provides two essential enhancements of the Ethernet: high accuracy/precision synchronization, and deterministic, reliable and low latency control data delivery. WR system consists of time and data master, network of WR switches, WR nodes. Reliability of WRN is based on four pillars: deterministic packet delivery, synchronization resilience, topology redundancy, and data resilience. The basic assumption of the WRN reliability is: WRN is functional if and only if it provides all its services to all its clients at any time. WR transmits two types of data: control data is transmitted with high priority (HP), and standard data is sent using the Best Effort (BE) mechanism - known from the TCP/IP networks. Characteristics of the control data are: sent in Control Messages, sent by Data Masters, Broadcast one to a lot, deterministic and low latency, reliable delivery. One time and data master may serve via a communication channel 2000 nodes distributed over $10 \mathrm{~km}$. Data redundancy is done via Forward Error Correction FEC. Re-transmission of Control Data is not possible. Forward Error Correction is served by an additional transparent layer. One Control Message is encoded into $\mathrm{N}$ Ethernet frames. The recovery of a Control Message is done from any of $\mathrm{M}(\mathrm{M}<\mathrm{N})$ frames. FEC can prevent data loss due to bit error and network reconfiguration. Topology redundancy is based on standard Ethernet solution, using rapid multi spanning tree protocol, with reconfiguration time shorter that $1 \mathrm{~s}$, typically tens of milliseconds. During 1s approximately 82000 Ethernet Frames are lost. Extensive research is carried on to extend the existing standards to prevent this huge data loss.

The solutions go into the following directions: to take advantage of the FEC mechanism, and to speed up the (R/M)STP protocol to eRSTP enhanced Rapid Spanning Tree Protocol). Together eRSTP and FEC give seamless redundancy which results in maximum loss of two frames. For 500 bytes long message with 288 byte FEC, the maximum reconfirmation time is approximately $2.3 \mu \mathrm{s}$. The features of eRSTP are: it provides a priori information - alternate and /or backup, limited number of topologies, drop only reception within VLAN except self-sending, take advantage of broadcast characteristic of control data, is going to be done in hardware. TRU Topology Resolution Unit is an universal and decoupled unit for topology resolution. It is a common firmware base for many different solutions. Currently two solution are considered: eRSTP (enhanced Rapid Spanning Tree Protocol) and eLACP enhanced Link Aggregation Control Protocol. WR switch consists of two layers S/W containing eRSTP/eLACP, and H/W containing TRU (RTU, Swcore and endpoints). 
The current status of WR system is as follows: Timingwise WR is working now with focus on data; there is an interest of standardization bodies - WR was presented to ITU$\mathrm{T}$ and IEEE; first deployment at CERN of WR timing and control network for AD; there is observed increasing number of applications; first commercially available WR switch is expected at the end of 2012 .

\section{Multi Pixel Photon Detectors}

Multi-Pixel Photon Counters for particle detectors: Multipixel photon counters (MPPC) are used in the particle detectors in high energy physics experiments. The research on MPPC is carried out in the team chaired by prof. J. Marzec at WUT. Multipixel (matrix) avalanche photodiodes are very sensitive and manufactured, for example, by Hamamatsu. They may operate in Geiger-mode, above some threshold value of supply voltage, with very high gain $10^{6}$. Active pixels are discharged from some higher to lower voltage, while the output current flow recharges the pixels. Histogram peaks, in pixel response, shows particular counts of photons. Peaks distance is determined by the electron gain. The main parameters of MPPC are: electron gain, junction capacitance, breakdown voltage, photon detection efficiency, dead time, dark rate, correlated noise. MPPC are used as PMT (photomultiplier tube) replacement. In comparison with PMT they have some advantages like: high gain and sensitivity, low price, small dimensions, are immune to magnetic fields, high reliability, moderate supply voltage around $100 \mathrm{~V}$, time resolution $300 \mathrm{ps}$. In comparison with PMT they have also some disadvantages like: high dark noise $10^{6} \mathrm{~s}^{-1}$, display correlated noise effects, have low active area (a few $\mathrm{mm}^{2}$ ). MPPC are used in the T2K experiment (Tokai to Kamioka) for research of neutrino oscillations. A measurement lab for mass testing of MPPC was constructed at IRE WUT, consisting of pulse generator, MPPC power supply, light hermetic case, temperature control, signal amplifier, multichannel amplitude analyzer. The system featured: compact design and modular electronics, high degree of automation, controlled measurement conditions and advanced data processing. There were applied two methods of dark rate evaluation: a direct method based on discriminator and scaler, and histogram based method. In the latter method the influence of correlated noise can be removed, while the first method is correlated noise sensitive. Correlated noise analysis is based on dark histogram. Correlated noise shows up as a difference between real histogram shape and Poisson distribution. Measurement of photon detection efficiency is based on light histogram, where the dark rate and noise must be taken into account.

High-Density Multi-Pixel Avalanche Photodiodes for particle detectors: Super-high density multi-pixel avalanche photodiodes (MAPD) are the next step in development of semiconductor matrix detectors with high gain for nuclear purposes. Structure of MAPD resides on $n$ substrate and $n^{+}$high doped layer. $\mathrm{P}$ type epilayer has $\mathrm{n}^{+}$microwells and is covered by $\mathrm{p}^{+}$contact layer. Photons impinging on the contact layer generate carriers, which are concentrated by $\mathrm{n}^{+}$microwells (pixels). Size of the pixels is $8 \times 8 \mu \mathrm{m}$. The comparison between traditional Silicon Photomultipliers (SiPM) working in Geiger mode (MPPC) with MAPD is as follows: density of pixels is bigger for MAPD and amounts tp $10000 / \mathrm{mm}^{2}$, gain is slightly smaller $10^{4}-10^{5}$, time resolution is $30 \mathrm{~ns}$. MAPDs were applied in the development of the Compass experiment in CERN. They had the following parameters: single detector size $3 \times 3 \mathrm{~mm}$, granularity $15 \times 10^{3}$ pixels $/ \mathrm{mm}^{3}$, gain $5 \times 10^{4}$, working voltage $90 \mathrm{~V}$, temperature stabilization. Detectors were coupled by Winston cone with WLS optical fibers.

Supersensitive multichannel pico-ammeter is under construction using FMC module, including PCB and firmware. The device is predicted to be used in a measuring system in a particle detector (HEP and astrophysical experiments), to cooperate with APD diodes, matrix detectors, and wire chambers.

\section{Superconducting Magnets}

Thermodynamics of superconducting magnets for accelerators. The magnets keep and focus charged particles in an accelerator and in the particle collider. Main dipoles are used to bend the beam in circular accelerators. The higher the magnetic field the higher energy particles can be kept on track. Main quadrupoles are used to keep the beam together, because of repelling forces in the charged particle bunched beam. Corrector magnets are used to preserve the beam quality. Interaction region quadrupoles are used to focus the beam to a waist at the interaction points. The higher is the gradient in the interaction magnets and the bigger aperture, the higher is the luminosity in the colliding beams focus. Superconducting magnets have replaced warm magnets because of energy efficiency and higher fields obtained in compact designs. Iron dominated magnets are limited by saturation at $2 \mathrm{~T}$. The state of the art large size DC magnets are now working in LHC and FLASH. The maximum field in the LHC dipoles, working at the temperature of super-liquid helium $1.9 \mathrm{~K}$, is over $8 \mathrm{~T}$. Three critical parameters determine the workspace of a magnet superconductor: current density $\mathrm{J}\left[\mathrm{A} / \mathrm{mm}^{2}\right]$, magnetic field $\mathrm{B}[\mathrm{T}]$, temperature $\mathrm{T}[\mathrm{K}]$. Above these critical values, which are property of the used superconducting material like $\mathrm{Nb}$, $\mathrm{NbTi}, \mathrm{NbSn}$, the magnet quenches, leading to beam dump. For example, the temperature of the superconductor may rise due to beam induced heat load, or too big current density in the coil. The machine and beam recovery after the dump may be quite time and effort consuming, depending on the cause of the loss (from a few $h$ to a few tens of $h$ ). Beam loss monitors (BLM) are used in accelerators to prevent beam loss and thus avoiding the full accelerator recovery processes. Only the beam cycling, filling, ramping and squeezing processes are repeated which lasts approximately for $2 \mathrm{~h}$ only. After these processes the collisions may be resumed. It is apparent that an efficient BLM leads to a substantial increase in the accelerator availability. Energy deposits in the accelerator magnets is a complex process because of a complex construction of the components and thus the heat dissipation paths.

The heat load in the LHC IR magnets is $10 \mathrm{~W} / \mathrm{m}$ and for the LHC upgrade will increase to $50 \mathrm{~W} / \mathrm{m}$. Precise thermal study of the magnets leads to: optimization BLM threshold 
settings, increase integrated luminosity, reduce quench number, optimize magnet cooling (D. Bocian, IFJ Kraków). The primary mechanisms of the heat load in the LHC magnets were fully recognized. Heat transport in superconducting magnet along the full path from the conductor to the cold source, including electrical insulation, was recognized and modeled. The network model includes all relevant parameters like material properties, detailed geometry of the construction, heat load profile, temperature margins, helium, cabling, magnets, etc. The built model, bases on thermal - electrical analogy, leads to a temperature map. The assumed equivalents are: temperature - voltage $\mathrm{T}-\mathrm{V}$, heat - charge $\mathrm{Q}-\mathrm{Q}, \mathrm{q}-\mathrm{I}$ heat transfer rate - current, $\mathrm{k}-\mathrm{s}$ thermal conductivity - electrical conductivity, $\mathrm{R}-\mathrm{R}$ thermal resistance - electrical resistance, $\mathrm{C}$ - C thermal capacitance - capacitance. The analogy of temperature rise is voltage difference. The analogy of heat diffusion is signal transmission in RC transmission line. The model was validated by measurements and simulated, and allowed for precise quench limit calculations. The model is used in CERN to study new magnets design parameters impact on magnet thermal performance.

\section{Photon Physics, Free Electron LASERs AND SYNCHROTRON LIGHT SOURCES}

Photon physics concerns the research of the properties of a photon as a boson, their mutual interactions, like gammagamma interactions, study of photon structure, virtual photons and interaction with matter at micro and macro scales, photon gas. Matter creation is a process inverse to particle annihilation, analogy to time reversal. Two mass less photons can be converted into two massive particles, like electronpositron pair, because of momentum conservation law. Energy conservation law prompts that the photons must be at least $1.022 \mathrm{MeV}$ (it is equivalent to the temperature of $10^{10} \mathrm{~K}$ ), which is soft gamma ray, to create an $\mathrm{e}^{+}-\mathrm{e}^{-}$pair. Creation of proton and antiproton pair requires $1.88 \mathrm{GeV}$ which is hard gamma ray (it is equivalent to $10^{13} \mathrm{~K}$ ). QUP - quark gluon plasma is created at the energy over $175 \mathrm{MeV}$, or temperature $2 * 10^{12} \mathrm{~K}$. Matter creation events and two photon effects take place in ultra-relativistic charged particle accelerator colliders. In these events, the beam of photons is radiated in very narrow cone (the flux is big) along the direction of motion of original particle. Production of baryonic matter out of photon gas requires high photon density and temperature. Above the threshold temperature the bosons and fermions inter-convert freely.

\section{POLFEL LASER}

POLFEL LASER. Polish Free Electron Laser $\left(4^{\text {th }}\right.$ generation light source) is planned in NCBJ Świerk (O. CholujDziewiecka). It will provide coherent EM radiation from a few $\mathrm{nm}$ (soft X-rays) to a few hundred $\mathrm{nm}(\mathrm{THz})$. The laser is scheduled to work in $\mathrm{CW}$ and pulsed regimes. The shortest soft X-ray pulses will be in the fs range. The shortest $\mathrm{THz}$ pulses will be in the range of ps. Peak power is around $1 \mathrm{GW}$ in the whole range of frequency spectrum. The machine is able to switch to high average power operation in the entire wavelength range. In the latter mode of operation it will be one of the most powerful sources of coherent radiation in this region. The final design predicts the following parameters for POLFEL: source - VUV-FEL, electron beam energy $>800 \mathrm{MeV}$, accelerating field frequency $-1.3 \mathrm{GHz}$, modes of operation - pulsed and $\mathrm{CW}$, repetition rate $-100 \mathrm{~Hz}, \mathrm{RF}$ gun superconducting via photoemission for a photocathode; radiation wavelength $<10 \mathrm{~nm}$, peak power $-0.2 \mathrm{GW}$, pulse energy $>10 \mu \mathrm{J}$, pulse width $<10 \mathrm{fs}$, average power $>10 \mathrm{~W}$, total length of linac around $300 \mathrm{~m}$. Polfel, typically to other FELs will consist of the following basic infrastructural parts: electron injector, electron accelerator, undulator, optical beam lines and experimental stations. The machine will be built in two stages, The first one is scaled down to one quarter of the above parameters. The linear accelerator of Polfel will be built using TESLA technology, which was used in the linac of FLASH and is currently used in the construction of the European Xray FEL. TESLA technology is also planned to be used in the future lepton collider for particle physics like the ILC project (International Linear Collider). Polfel undulators will enable generation of light with different states of polarization - linear and circular. Transport of the laser beam is predicted to nuclear reactor Maria for common experiments involving laser and neutron irradiations. Polfel is expected to be open for transnational access programs within the UE collaboration and financing.

Polfel will complement the capabilities of conventional lasers by extending the available generated spectrum. Polfel will provide crosswise and longitudinal coherence light, which is impossible for the synchrotron $\left(3^{\text {rd }}\right.$ generation light source). Polfel will provide higher intensity by several orders of magnitude and pulse duration three orders of magnitude shorter. Polfel will enable the development of fundamental and technological research, in this region, in physics, chemistry, biology, material engineering etc. These experiments to be effective seek for bigger coherence of light (transverse and longitudinal) and shorter light pulses to avoid excessive heating and to make the reactions more adiabatic and bigger intensities to enable deeper effects. Predicted experiments for Polfel will include, but will not be limited to the research on: photon physics, properties of light and its interaction with matter, including the highly excited states of matter investigated now in plasma physics and astrophysics; molecular dynamics and transient states in excitations and chemical reactions; the structure of large biological molecules and systems of new organic semiconductor and magnetic materials; surface modification by irradiation, determination of the spectral signatures of materials in different wavelength ranges.

Superconducting RF electron Gun for high average power. FEL Superconducting electron gun for $\mathrm{CW}$ operation of superconducting linacs is under design by a collaboration consisting of TJNAF, NCNR, HZB, HZR and DESY (R. Nietubyć, NCNR, Świerk). The purpose is to build a gun suitable for building of tunable $\mathrm{CW}$ free electron laser (high average power FEL) based on superconducting $\mathrm{Nb}$ cavities linear accelerator. The work stemmed from plasma physics laboratory and European FP6 project CARE - Coordinated Accelerator Research in Europe. Now the first e-gun is available with 
lead photocathode. Last year progress include: e-gun tests in HOBICAT (horizontal bi-cavity test facility), application of new cavity with plug in and inclined back wall, design of new lead deposition system, emittance studies of the new design. The new design use $\mathrm{Pb}$ as a superconducting emitter, in a form of lead thin film spot for photocathode $(400 \mathrm{~nm}$ thickness). $\mathrm{Pb}$ is deposited in UHV arc vacuum system with droplets filtering and adhesion optimization. Ions emission is from a small explosive spot. This spot is a local explosion decompression and ejection of neutrals and ions. High ions fraction enables a coupling to electrons and driving with the $\mathrm{H}$ field. The deposited $\mathrm{Pb}$ spot was annealed with a laser beam for better quantum efficiency $\mathrm{QE}=3.3 * 10^{-3} . \mathrm{Pb}$ is a superconductor of the first type with the following parameters: critical field $\mathrm{H}_{\mathrm{c}}=80 \mathrm{mT}$, critical temperature $\mathrm{T}_{c}=7.2 \mathrm{~K}$, work function $\mathrm{W}_{\mathrm{e}}=3.95 \mathrm{eV}$, quantum efficiency $10^{-5}-10^{-2}$ in the range of photon energies $4 \mathrm{eV}-7 \mathrm{eV}$. Fully superconducting injector avoids normal conducting materials in the cavity, keeps simplicity similar to $\mathrm{Nb}$ back wall gun, leads to reasonable quantum efficiencies QE. To facilitate transport of samples between the laboratories the cavity was drilled and provided with a plug. Only the plug was coated with $\mathrm{Pb}$ spot. Baseline tests for cavity with and without a plug give the same results for the field maximum of around $53-54 \mathrm{MV} / \mathrm{m}$. With $\mathrm{Pb}$ coated plug DESY II lab demonstrated reasonable finesse of $\mathrm{Q}_{\mathrm{o}}>4 * 10^{-9}$ up to $33 \mathrm{MV} / \mathrm{m}$. With the tests at BESSY the experience is accumulated with the photocathode and with the LLRF cold tuner. Back wall symmetrical inclination of $8^{\circ}$ gives improvement in the slice emittance. The aims are the following parameters of the SC injector: $1 \mathrm{nC}$ and $1 \mu \mathrm{m} * \mathrm{rad}$ at $50 \mathrm{~Hz} ; \mathrm{QE}>2 * 10^{-3}$ at $213 \mathrm{~nm}$ and $\mathrm{QE}>2 * 10^{-5}$ at $258 \mathrm{~nm}$; $\mathrm{E}_{\text {peak }}>60 \mathrm{MVm}^{-1}$ at $\mathrm{Q}_{\mathrm{o}}>10^{10}$ with $\mathrm{Pb}$ spot.

Electron beam stabilization in free electron laser: The free electron laser to shine a stable and coherent beam requires an electron beam of very high quality. A research on digital feedback system for direct electron beam parameters stabilization is carried on by the LLRF team of the NCBJ, Świerk (J. Szewiński) in cooperation with DESY. Classical LLRF feedback system stabilizes only the amplitude and phase of the high power RF field. More stable RF field does not directly cause the beam to be more stable above some stability threshold. Higher gain value in the feedback loop amplifies the noise, which degrades the beam. Beam stability in terms of $\mathrm{dE} / \mathrm{E}[\%]$ i.e. energy dispersion, as a function of feedback loop gain, has an optimal value for gain coefficients around 10-20. The basis of energy feedback of the electron bunch is similar to the action done by a prism in optics. Dipole magnets act as prisms. Energy dispersion of electrons in a single bunch is changed to their different path lengths. Red electrons (less energetic) are lagging behind the blue electrons due to dispersion. The bunch compressor equalizes the delays by delaying blue ones and speeding the red ones. The bunch has much bigger quality after the compressor to give better photon beam in the undulator. Arrival time of electron bunch and its length is measured by Bunch Arrival Time Monitor BAM. BAM is a photonic device where amplitude of laser pulses generated by the reference synchronization system (MLO) is modulated in electro optic modulator EOM by the arrival time of the travelling bunch. The amplitude of the bunch which arrives in due time is the same as the amplitude of the unmodulated laser pulses, due to zero-crossing. The signal from photo diode is sampled using two ADCs, with clock signal shifted in phase. Bunch compression is measured by the pyro-electric detector PYRO. PYRO is located after the bunch compressor and the bunch comes through it. Amplitude of generated electric pulse is proportional to the compression rate of the bunch. The system was installed at FLASH laser.

LLRF system for POLFEL laser: POLFEL accelerator, to be built at the NCBJ in Świerk, is expected to have the following parameters, which are important for the LLRF (low level RF distributed control and measurement system): operation modes are either $\mathrm{CW}$ or long pulses $\mathrm{t}>100 \mathrm{~ms}$; superconducting electron source; superconducting $1.3 \mathrm{GHz}$ accelerating structures of 4GLS or TESLA type criomodules; IOT - inductive output tube as an RF amplifier. Digital LLRF feedback system is predicted with I-Q field detection. Due to difficulties in field phase measurements, better results can be achieved by detection of In-phase and Quadrature components of the cavity signal. The I-Q may be detected digitally. Sampling with frequency four times higher than the IF (intermediate frequency - after down-conversion) gives the I and $\mathrm{Q}$ values directly. Analog RF modulation is simple, there are ready to use vector modulator integrated circuits, with mixer and phase shifters in a single chip. In a digital RF modulation one DAC per channel is saved. However, RF analog part is more complex with needed band pass filters. Two control systems are options: GDR - generator driven resonator and SEL - self excited loop. GDR features are: frequency is fixed, better for pulsed operation if the detuning is small, fast predictable rise time, better to perform vector sum control. SEL features are: tracks the resonant frequency, better for $\mathrm{CW}$ operation, start up may be slow, better for single cavity control. The choice for POLFEL is (J. Szewiński, NCBJ, Świerk): feedback loop digital feedback based on vector sum with $\mathrm{P}$ and I gains; field detection - digital I-Q demodulation; analog RF modulation; control method - SEL with cavity detuning compensation, optional GDR mode. The hardware for POLFEL LLRF may base on the proven SIMCON VME technology. The control environment would be EPICS.

\section{SOLARIS SYNCHROTRON}

Jagiellonian University in Kraków is building synchrotron based national light source (P. Tracz, UJ, Kraków, National Synchrotron Radiation Centre). Diagram of the source is presented in fig. It consists of linear injector (e-gun and liniac working at $3 \mathrm{GHz}$ frequency), storage ring and beam lines. The first beam line U1 is predicted for soft X-ray spectroscopy. The photon energy range is $200 \mathrm{eV}-1.5 \mathrm{keV}$. Radiation source is undulator. The used experimental techniques are: PES photoelectron emission spectroscopy, XAS - X-ray absorption spectroscopy, and RIXS - resonant inelastic X-ray scattering. The storage ring parameters are: $1.5 \mathrm{GeV}$ energy, $500 \mathrm{~mA}$ current, circumference $96 \mathrm{~m}$, horizontal emittance around $6 \mathrm{~nm}$ $\mathrm{rad}$, coupling $1 \%$, overall lifetime $13 \mathrm{~h}$. Compact lattice in the storage ring consists of 12 double bend achromats (DBA) 
cells, $12 * 3.5 \mathrm{~m}$ long straight sections, out of which 10 are for insertion devices. The magnets have combined functions for compact optics and include gradient dipoles and quads with integrated sixtupole.

Solaris synchrotron uses Tango Distributed Control Environment (P. Goryl, UJ, Kraków). Tango is for integration of equipment; providing, collecting and presenting data; logging data for future reference; accessing hardware in a standard way; managing the control system; configuring fast control loops like setting parameters and setpoints. Tango is not suitable to do fast feedback and is not real time by itself. Tango uses tools: Jive - device configuration, Astor - system deployment and management, Pogo - code generation, Sardana - scanning and macro running, JDraw - rapid GUI development, Taurus Library, ATK, and other. TANGO kernel contains API and TCP/IP CORBA and is an interface between configuration, GUI application, archive and devices. Tango is used by many synchrotron labs: ALBA. DESY, SOLEIL, ELETTRA, MAXLab, FN HML FRM II, and ESRF.

\section{XiX. Thermonuclear Technology and Plasma RESEARCH}

Ultimately hot plasma is researched, also using photonic methods, for a few different purposes, including: properties of distinct state of matter, space and astronomical object entity, constituent of solar atmosphere, an environment of thermonuclear fusion. The latter area is combined with the trials to build a thermonuclear power plant in the future. Depending on the financing, such a plant may be built during the next five decades or so. The way to a thermonuclear plant utility leads via the research on the development of energy efficient tokamaks and stellarators.

JET, a precursor of ITER. JET - Joint European Torus, realized as a tokamak, is an experiment which is expected to expand into ITER and then into thermonuclear energy utility. A lot of photonics and electronics equipment is realized around this experiment with the participation of teams from IFPiLM (Institute of Plasma Physics and Laser Micro-fusion in Warsaw, EURATOM), in cooperation with NCBJ Świerk, WU and WUT Universities. JET is realized inside the EFDA (European Fusion Development Agreement). JET wall materials are beryllium and tungsten. It features the in-vessel toroidal magnetic field up to 4T. The circular plasma current is over 3MA. The D-shape plasma current is around 5MW. Lifetime of the plasma is 20-60s. Auxiliary heating comes from NBI - up to $34 \mathrm{MW}$ and ion cyclotron resonance heating up to $10 \mathrm{MW}$. A typical tokamak plasma consists mainly of electrons and deuterons together with a small concentration of impurities. These impurities come mainly from interaction between the plasma and the vessel wall. Once impurities enter the plasma they become highly ionized and radiate energy thus cooling the plasma. The radiation can be lost by Bremsstrahlung and characteristic X-ray radiation. The major JET tokamak chamber is densely surrounded by diagnostics. Our team works on $\mathrm{KX} 1 \mathrm{X}$-ray crystal diagnostic spectrometer. The larger is the spectrometer the better is the energy resolution $\lambda / \mathrm{d} \lambda=$ $(\mathrm{R} / \mathrm{dx}) \tan \theta$. The spectrometer is operated in so-called Johann geometry with the Rowland circle radius of about $12 \mathrm{~m}$ giving an excellent resolution $\lambda / \mathrm{d} \lambda=2 \times 10^{4}$. The plasma rotation velocity is around $10 \mathrm{~km} / \mathrm{s}$. The soft $\mathrm{X}$-ray photons $(2-12 \mathrm{keV}$, which is the measurement range) are reflected by a germanium crystal (of 220 cut and of crystalline constant $4 \AA$ ) cylindrically bent with a radius of curvature of around $25 \mathrm{~m}$. The line of sight of the X-ray crystal spectrometer diagnostic is full cross section of the plasma channel. The main task addressed by the high resolution X-ray diagnostic (KX1) is probing the 100 million degree core of the JET plasma. The system is looking for the contamination of the plasma by tungsten (and $\mathrm{Ni}$ ) released from the newly installed ITER-like wall. The highresolution X-ray crystal spectrometer is expected to measure: impurity concentration, ion temperature and toroidal rotation velocity (rotation frequency $25-110 \mathrm{krad} / \mathrm{s}$ ). The measurements are based on the $\mathrm{Ni}^{+26}$ resonance line at around $7.81 \mathrm{keV}$, $\mathrm{W}^{+46}$ line at $2.4 \mathrm{keV}$, and continuum radiation. Once tungsten erodes from surfaces and penetrates the high temperature plasma a plentitude of tungsten charge states may be produced across the confined plasma profile. For JET plasma conditions, the expected charge-state distribution spread around Ni-like $\mathrm{W}^{+46}$ in the hot core, requiring sophisticated and specialized detection and analysis systems for line identification (from $\mathrm{W}^{+44}$ to $\mathrm{W}^{+50}$ ). In order to measure the tungsten impurity concentration in the new ITER-like JET configuration, the upgraded spectrometer will operate with an additional quartz crystal (of cut 1011 and crystal constant $2 \mathrm{~d}=6.7 \AA$ ) mounted in 2009 and sharing the same 266mm diameter beamline. The quartz crystals are used for the reflection of low energy $\mathrm{X}$-ray photons emitted by $\mathrm{W}^{+46}$ at $2.4 \mathrm{keV}$. The higher energy range of the $\mathrm{W}^{+46}$ spectra can be measured by means of the Si crystal which has been provided together with a new quartz crystal and can be curved and installed at a later time. The measurements are based on Bragg spectroscopy: $\mathrm{n} \lambda=2 \mathrm{~d} \sin \theta, \mathrm{E}=\mathrm{hc} / \mathrm{n} \lambda$, for the $\mathrm{n}$-th order of reflection and $\mathrm{SiO}_{2}$ crystal. $\mathrm{E}(\mathrm{n}=1)=[2.3 ; 2.5] \mathrm{keV} ; \mathrm{E}(\mathrm{n}=2)=[4.6 ; 5.0]$ $\mathrm{keV} ; \mathrm{E}(\mathrm{n}=3)=[6.9 ; 7.5] \mathrm{keV}$. With usual positive sensitive detectors it is not possible to distinguish between different orders of reflection. Position sensitive detectors with energy resolution allow us to distinguish between different orders of reflection. By application of the distinguishing procedures the measurement system provides: more data; pure X-ray spectra for $\mathrm{W}^{+46}$; more precise information on tungsten contamination, ion temperature and rotation velocity of the JET plasma; Z effective information from continuum; ITERrelevant diagnostic system.

Gas Electron Multiplier GEM plasma detector system: The plasma diagnostic system researched by a team from IFPILM (IPPLM), UW and WUT, uses GEM (gas electron multiplier) detectors. They have to be precisely calibrated. The particular structure of choice is T-GEM (triple GEM) of optimal voltage and space distribution. The size of gas detector is $100 \times 200 \mathrm{~mm}^{2}$. Hole sizes are $70 \mu \mathrm{m}$, while hole separation is $140 \mu \mathrm{m}$. The filling gas is: $\mathrm{Ar} 70 \%+\mathrm{CO}_{2} 30 \%$ flowing at a rate around $30 \mathrm{ml} / \mathrm{min}$. T-GEM structure with charge gain consists of transparent cathode and a window permeable for $h \nu$ photons, three layers of GEM and conducting pixelized or stripped anode. The measurement is energy and position resolved. The 
tested windows for soft X-ray photons were: $12 \mu \mathrm{m}$ mylar with $0.2 \mu \mathrm{m} \mathrm{Al}$, or $5 \mu \mathrm{m} \mathrm{W}, 12 \mu \mathrm{m} \mathrm{Ni}$. Detection efficiency was measured for various windows. A GEM detector is the first module of the measurement chain for X-ray detection of tokamak plasma. The space charges which are collected on the strip plane generate current anode signals for 256 channels. Multichannel system should be calibrated to uniform all channel characteristics. The succession of signal generation in a GEM is: photon conversion to electron, avalanche multiplication of charge, drift and diffusion of electron via the induction gap, discharge and generation of the anode signal. GEM detectors are calibrated using signal from $\mathrm{Fe}^{55}$. The electronics is calibrated for all 256 channels to equalize the individual channel amplification. Calibration charge type includes cluster and strip. Calibration parameter type includes gain charge and mean charge. A general assumption for calibration procedure is as follows: for regular charge distribution a parameter value for each channel is equal to the mean value for each channel. Thus, the calibration coefficient is equal to the inverse of normalized parameter. Fast electronics based on FPGA circuits is used for energy estimation and creation of real time histograms.

Plasma Focus PF-1000 experiment: A new generation of data acquisition system for the plasma fusion experiment PF1000 is under preparation. The experiment is located at the Institute of Plasma Physics and Laser Microfusion in Warsaw. The plasma focus device is a compact powerful pulsed source of intense radiation including neutrons, X-rays, optical and particle beams. The radiated spectrum depends on the temperature and the used sample material. The amount of stored energy in the discharge capacitors exciting the device is over $1 \mathrm{MJ}$. The experiment is a convenient local test bed for some measurement, diagnostics, procedures and hardware designed finally for implementation at the European JET and ITER experiments. PF-1000 is used to diagnose plasma dynamics and stability for future prototypes of specific high temperature plasma reactors. Plasma focus device consists of anode and cathode separated by an insulator. The hermetic chamber is filled with deuterium under pressure optimized for particular experiment. The collector is a part of the power supply for $\mathrm{PF}$ - which are high power capacitor banks. The plasma creation event is triggered by a laser pigtailed with an optical fiber. Now, the experiment features a number of old analog diagnostic solutions, over 10 of them, which are successively replaced by modern FPGA-DSP-CPU and photonics sensing and transmission based multichannel systems.

\section{Open Hardware Repository}

Open hardware repositories are increasingly frequently supported by large research organizations financed from public funds. The process is similar to the analogous actions undertaken in the area of publications of the research results. Contemporary electronics and photonics contains now more IP intellectual property than ever before. And this effort to generate this IP is financed publically. One of the current development trends in the photonic and electronic functional systems for research and/or industrial applications relies on very low cost application tailored solutions, making the hardware virtual and enabling All designs in open hardware repositories. This may change considerably the approach to photonics and electronics design, usage and marketing. This situation may be compared to the situation on the open publishing market, where some big and influential i.e. leading research institutions make the results of their work open access rather than publishing them in high-rank closed archival journals. The designs from the open access repositories may be used by manufacturers freely and after fabrication marketed of their own. An absolute leader in this approach is CERN, but there are more and more followers. Here we present a few open access hardware designs origination in the PERG/ELHEP and other associated Research Groups. The designs are destined for HEP experiments, photon physics research, astronomical and space equipment, plasma research, biomedical engineering applications, etc (dr W. Zabołotny, dr G. Kasprowicz).

Features of open access hardware: Most FPGA based systems, especially these used for data acquisition functions use a bus (typically VME bus, but more modern ones use ATCA bus) to provide access to internal registers. The VME or similar solutions require to use expensive components like VME crates, VME controller, etc. If we need to debug the VME board more thoroughly, with a scope, we need yet another VME extended board, which still does not allow for easy access to the vertically positioned board. A question arises: Can we do it simpler? The design goals are to do it simpler and cheaper. Low cost interface is very convenient, which could be easily connected to the development machine, which usually is a laptop. Such solution may also be used in the final system. The outside PC is connected with the embedded PC. This solution provides easy development of FPGA based system via the JTAG access for configuration and debugging. It gives also a convenient possibility to work with the board alone, completely without the crate. A typical development machine should contain several basic and universal interfaces and make them available for the system design purposes.

Common platform - Ethernet, Linux, FPGA: The Ethernet is usually needed to connect to the local network/Internet. Use of typical TCP/IP protocols would require significant resources in the interface, but the use of nonstandard protocols may cause software compatibility issues. The PCIe interface is available in ExpressCard slot. Only one board may be connected. The usage of hardware PCIe bridge in the interface is necessary, and only short cables may be used. The advantage of using the USB is that multiple boards may be connected via a hub, the cables are cheap and it seems to be the optimal solution. How to connect an arbitrary development board to the USB? In the case of direct connection - a PHY chip is required, where a relatively complicated software support is needed for USB compatibility including enumeration. A connection via USB bridge is significantly cheaper with the most popular USB bridges like Cypress, FX2LP, FTDI. The functionalities which are offered by FTDI FT2232H, including multiple operating modes, are: USB to FIFO transfer data rate practically up to $8 \mathrm{MB} / \mathrm{s}$, single channel synchronous FIFO mode for transfers practically up to $30 \mathrm{MB} / \mathrm{s}$, CPU style FIFO interface mode simplifies CPU interface design, Dual 
multi-protocol synchronous serial engine (MPSSE) is used to simplify synchronous serial protocol design like USB to JTAG, I2C, SPI or bit-bang. The following selection of modes in FT2232H is available: local bus emulation (the slowest solution), parallel FIFO mode (the fastest solution, however single channel), asynchronous FIFO where the second channel may be used as JTAG, ChipScope, Impact. System architecture is as follows: USB, FT2232H, channels A and B, FPGA (and JTAG access), local bus and fast JTAG. Commands and data are transferred through FIFO, therefore encapsulation technique is used. Interfacing with the development machine includes several ways: directly from the Python scripts, using the module of pyusb; from the $\mathrm{C}$ code using the library libusb; from Matlab scripts via TCP/IP connection using the server implemented in $\mathrm{C}$ with libusb. The described interface was successfully used in development of control and readout system for GEM detector for KX1 detector in JET experiment. In the final system, the interface is connected to the USB port of embedded PC and is used to provide slow control, diagnostic, configuration functionalities supporting the main PCIe based data transmission channel. Now the solution works as a low price efficient way to control the local bus in the FPGA based systems. Usage of synchronous FIFO improves the transmission parameters of the interface at a cost of slow JTAG channel. Then, ChipScope may be used for debugging. Usage of USB 3 bridge is expected to improve all system parameters.

Hardware - software co design: Hardware - software codesign paradigm is one of the issues of contemporary functional systems. Now the advanced photonic and electronic systems integrate heavily hardware and software layers. Hardware and software are configurable. Combination of hardware and software layers requires proper communication between them. Prototyping of a new photonic and/or electronic system requires significant redesign stages. Multiple repetitions of the redesign stages are costly and time consuming unless the hardware and software are not widely configurable. Software components of the system, including device drivers, user space application, etc, may be developed in parallel as soon as hardware specification is ready. Such approach may expose some problems related to the hardware design. These issues appear during attempts to write the code, when a skilful programmer detects obvious inefficiencies. Thus, a full testing of the system is not possible until the hardware is ready. At the early stage of development it may be useful to verify functionality of the design using Virtual Hardware (VH). VH environment is used to develop ne software, like emulators and simulators, including mobile phones. Typical simulators emulate standard platforms. Description of digital hardware in HDL, including Verilog or VHDL, makes the hardware simulable. Efficient simulators for this purpose include: Modelsim, GHDL, Icarus Verilog. It is inefficient to emulate the whole VHDL model of the system (including CPU, program, data memory). An interesting solution is a behavioral, optimized for simulation, model of the whole PC system (or only a CPU). Such a model may be run in the hardware simulator. Thus, the task is software co-simulation with hardware simulators. An effective approach, for simple programs, is when the tested software communicates with the simulated hardware, a if it were with the real one. The methods include: simulated bus controller allows Python programs to access local bus in FPGA; simulated UART allows the simulated hardware to exchange data with Linux pseudoterminal pts device, etc. Another method bases on software simulator emulating PC system and hardware simulator emulating real hardware, which is called HW/SE cosimulation, where precise time synchronization is required for both systems. Two way system response is possible. Hardware responds to requests from software simulator. Hardware simulation is then run for time periods between interaction with hardware. Hardware also reacts to external stimuli and generates asynchronous events like interrupts for the PC simulated with software simulator. Time synchronization in the universal case requires full integration of the HW simulator kernel with the SW simulator. The HW and SW simulators should be tightly coupled like in QUEMU System C by GreenSocs. Another solution is OVP (open virtual platform). QUEMU is a simulator enabling additions of own HW, like x86 32 and 64bit, ARM, PPC, MIPS and other ones. QUEMU was used successfully for development of software part of the GEM readout system, for testing of USB based communication and of network data distribution. Some models of own HW were implemented including: high speed ADC, AES256 cryptographic accelerator, experimental network adapter supporting BM DMA and SG buffers. VH may be a tool allowing to decrease gravely time and cost of HW SW co-development. However, no ideal solution as yet exist. Appropriate tool must be carefully chosen for particular application. QUEMU may be a good solution if the HW can be described in C. API is sparsely documented and often changing.

Industrial standards and FMC carrier based systems: A number of various hardware designs and hardware - software codesigns are under preparation to be available in the open hardware repository (PERG/ELHEP Research Group at ISE PW). These include: Low cost FMC carrier board with embedded ARM processor and fast optical I/O, Linux running module on Power PC embedded processor with FPGA interface, Multichannel pico-ammeter FMC module, group repository environment, combustion engine parameters recorder, universal image acquisition system on FPGA, acceleration artificial intelligence algorithms with reconfigurable computers, handheld distributed measurement system, multichannel digital oscilloscope for universal measuring platform. A number of these design use FMC carrier with ARM processor and Linux OS.

The main idea is to build an open source platform basing on FMC standard, for computing and measurement applications. The existing competing platforms are as follows and have some drawbacks:

- CPCI and VME - they have unpredictable latency and low bandwidth;

- uTCA, ATCA - high cost, expensive controller, complicated packet based interfaces, good for computing data and communication, not really suitable for low latency data processing and fast control systems; 
On the other hand the FMC standard (ANSI for FPGA Mezzanine card) maximizes data throughput, minimizes latency, reduces FPGA design complexity, minimizes system costs, reduces system overheads. FMC standard supports VME, VPX, VPX REDI, CompactPCI Express. Cooperates with ATCA, AMC, PCI and PCI carriers, PXI and PXI express carriers. A family of high speed connectors for IO mezzanine modules are available. It supports up to $10 \mathrm{~Gb} / \mathrm{s}$ transmission, and single ended and differential signaling up to $2 \mathrm{~Gb} / \mathrm{s}$. Has numerous IOs available. The electrical connectivity of the IO mezzanine module is provided by high speed connector which supports a wide range of signaling standards, supports system configurability of IO functionality, provided FPGA intimacy. The mechanical properties of the IO mezzanine module are: minimal size, scalable from low end to high performance applications, has conduction and ruggedized support. The following questions are addressed during the design (G. Kasprowicz, PERG Group, ISE PW). How to enable low latency data transfer between FMC modules, which is critical for DSP based on correlation? How to obtain low cost, which is essential for multichannel systems? How to obtain high speed serial transfer capability? How to obtain simplicity of parallel IO modules? How to obtain non-limited scalability? And finally, how to obtain it all in a single design?

Parameters of the FMC carrier. FMC carrier was designed featuring: 36xFMC sockets, PCIe Gen@(5Gb/s), low jitter clock distribution network, White Rabbit IEEE 1588 enabled, standard main board with 4X86 cores, maximum 46 FPGA chips. The specific FMC carrier features are: modularity, up to 18 modules in $2 \mathrm{U} 19$ " case, not defined logical interface, just 38 bidir LVDS lines, up to $24 \mathrm{~Gb} / \mathrm{s}$; any type of interface is possible to implement; star type connection, deterministic behavior; latencies may be as low as a few ns; low cost measurement modules; they may be even passive; central processing unit with storage; PCIe Gen II 5Gb/s non blocking switch; centralized low jitter clock management; centralized FPGA configuration; I2C, SPI, JTAG management interfaces; intelligent module detection; dedicated FMC sockets for DSP accelerators or 10Gbit/s connection; embedded strong multicore x86 with Linux. Recently developed FMS modules embraced, for example, $4 \times 125 \mathrm{Mhz}$ oscilloscope featuring 16 $\times$ ADC + SDRAM + FPGA; quad time to digital converter and fine delay lines, featuring 8 core DSP + FPGA. The computing and DSP power of FMC system is: 10XFPGA 23k slices, 147k logic cells, 180DSP slices, maximum $500 \mathrm{MHz}$ each + 16x SDRAM 1Gb; plus 34x FPGA each of FMC; two dedicated FMC slots for 8 core FP DSP, 2GbEth ports' 21 port PCIe switch connecting FPGAs, FMCs and Power PC. The FMC carrier - ITX main board features: ASUS 2 core $\mathrm{x} 86$, FPU and network accelerator; PCIe $2 \times 4$ lane GEN2 switch, $1 \mathrm{xGb}$ Ethernet interface, USB local bus converter FPC' MiniITX format. The exemplary applications of the FMC system include:

- 256 channel, 100MS/s data acquisition system each with online processing for HEP with aggregated $256 \mathrm{~Gb} / \mathrm{s}$ of the on line data processing throughput;

- 2048 channel slow speed DAQ;
- Low power computing platform - 16 modules equipped with 8 quad core ARM processors running at $1 \mathrm{GHz}$; up to 1TIPS of peak computing power available in PC-size box, with $4 \mathrm{k}$ Euro budget, consuming $150 \mathrm{~W}$ of supply power;

- 16 ADC modules plus 16 quad DSP modules for advanced radar applications;

The new hardware solution of the system features: it is based on recent large Kintex 7 FPGA circuits, which results in fewer chips onboard, fewer connectivity problems and resources used for communication; 6 carrier slots; up to 12 HPC dual with FMCs addressing the clock issues; 4U case with improved cooling; fully symmetrical architecture with no dedicated controller FPGA; each carrier may perform controller function; backplane with PCIe switch and cross-point 48 channel switch up to $5 \mathrm{~Gb} / \mathrm{s}$; use of standard ITX PC board; USB and small FPGA for JTAG and slow control; optimized for HEP applications like: LLRF system for accelerators and free electron lasers, in particle detectors and BPM - beam position measurements. The project is fully opened and done in cooperation with the Brazilian LNLS laboratory.

Real time Embedded Systems for DAQ. A research work on real-time performance of Linux based embedded system is realized by a team in the Institute of Aviation in Warsaw (R. Rybaniec). The aim of the work are cheap airborne applications. It is to be noted however, that Linux is not inherently a real time system. Real time operation systems, software environments or isolated programs, algorithms and procedures must guarantee a deterministic response within very strict time constraints. There is also a number of terms around this notion like soft-real-time, near-real-time and finally hard-real-time (or real-real-time). In the soft-real time system, after crossing the deadline, usefulness of the system response is degraded but still may be of attention. In the hard-real-time, missing deadline means full system failure, with no exception. Real time means fully deterministic, synchronous. Parameters of real-time systems are latency and jitter. Latency is time period between an event occurring in the system and end of processing of this event by this system. Jitter is a variation in periodic timing in the system. Jitter should be as small as possible in the real time systems as it introduces uncertainty. Linux is popularly used in embedded systems. Embedded systems are popularly used in construction of real time systems. Linux is used in embedded systems because: is a versatile, thoroughly checked, and very good application base, is popular and used by large community also professional, is characterized by out of the box operation, free of charge and open source with ease of configuration. Linux is not a real time system, even not a near real time. It is a general purpose OS sharing resources in a "fair" manner, optimizing resources for maximal throughput and maximum average performance. Maximum, but set, latency is never guaranteed. It is a typical best effort type system. But, using special methods like nano-kernel approach and real time shells, it may be turned to near real time. There are a lot of purposes to do this. Such an open source near real time OS may service demanding data acquisition systems for airborne applications like for gyrocrafts. Such systems base 
on cheap PCBs containing ARM processors and cooperating with numerable RF and photonic teledetection sensors. Linux Xenomai uses nano-kernel approach with domain concepts, kernel is low priority domain and fully preemptive. Improved preemptiveness of Linux Preempt_RT kernel relies on that the preemption is active in kernel mode and also critical section are preemptive. IRQ service routines are threading with preemption in ISR with priorities. Both Xenomai and Preempt_RT approaches significantly improve real-time performance of Linux. Xenomai shows better performance while in Preempt_RT less changes are needed. Xenomai enabled kernel proves to work well in data acquisition system (SPI $400 \mathrm{~Hz})$.

Open hardware projects: The projects listed below are currently under realization in the PERG/ELHEP ISE WUT Research Group. Most of the results are intended to be available in the open hardware repository.

- Implementation of PCI Express bus communication for FPGA based data acquisition systems: communications core is based on mixed Verilog/VHDL; Linux OS and device drivers;

- Multisensor, low-cost measurement LAN for use in personal cars, cooperation with temperature, humidity, vibration and acceleration low power sensors;

- Local positioning system for use inside buildings, to replace lot of RFID readers and solve the screening effect for GPS, based on White Rabbit, low-cost realized on FPGA, method based on triangulation and trilateration;

- Mobile measurement system based on a smart cell phone (iOS and Android) with capacity to read numerable distributed sensors located at the user promontories, and with user friendly integrated and interactive display;

- Measuring system for hybrid power plants including combination of various generators: wind and solar;

- Detection system for car driver physical status including: closed eyes, movements, some physiological parameters, tracking drivers face, anti sleep alarms, lane departure;

- Artistic design table controller using display screen modules, organization of the array of touch display screens;

- Mult-sensor ultimately low-power point with energy harvesting;

- Control system for omni-directional mobile robot wheeled and quadrocopter;

- Real time omnidirectional camera with parallax mitigation techniques and image alignment and stitching;

- Interactive whiteboard with laser pointer and CMOS camera, standalone device;

- Multichannel ultrasonic/photonic data acquisition system;

\section{INDUSTRIAL STANDARDS}

A marriage between FPGA and ARM processors (alternatively PowerPC CPU or software based CPU MicroBlaze) on a single, universally equipped $\mathrm{PCB}$ with relevant I/Os (including fiber and/or wireless) and $\mathrm{ADC} / \mathrm{DAC}$, results in a very powerful application system. Such a system was used for building an extended network of sensors, for control of hybrid power station (wind turbines and photovoltaic), environmental

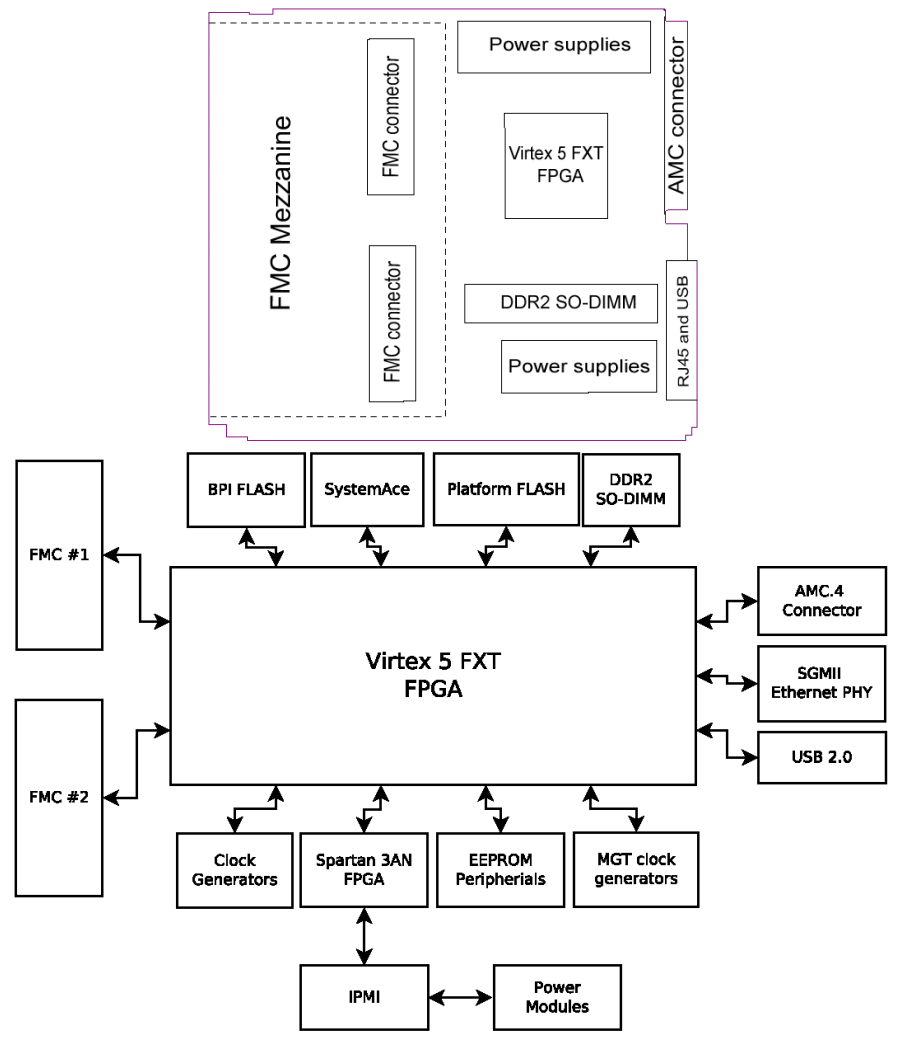

Fig. 1. A $\mu$ TCA carrier board and FMC Mezzanine (ADC daughter) for fast EVM.

monitoring, and many other. AD9980 circuit on such a board provides all HDTV modes. CH7301B circuit makes the board compatible with the CVI and other video and control standards (RS, VGA, DVI). The software is readily manufactured in the FPGA vendor environments, like the vga2dvi converter module, and many others. The basic assumption is to make the system multichannel and flexible for different kinds of sensors, including RF and optical. Such a universal mother PCB may be done experimentally, with test architectures containing FPGA, CPU, memory, internal multi-gigabit bus, GbE optical links and other I/O ports. However, a number of FPGA and CPU vendors offer Evaluation Platforms of quite universal architecture and all the necessary key components onboard. One of the frequently used examples is V-5FXT FPGA ML507 by Xilinx. The system is conveniently compatible with the ISE Design Suite software environment. Such solutions speed up considerably the design of practical functional blocks of various photonic and electronic systems like, in our case, multi sensory networks, video systems and others.

The $\mu$ TCA standard, a miniaturized derivative of ATCA (advanced technology communication architecture), has recently been used not only for telecom but also for the development of measurement and control systems in high energy physics experiments, astronomy, biomedical engineering, free electron lasers, accelerators and large research infrastructure. $\mu \mathrm{TCA}$ format was officially chosen by some large scale photonics infrastructure like European X-FEL laser, FLASH laser etc. One of the subsystems made in this technology is the upgrade of the Bunch Arrival Time Monitor for the large E-XFEL 
machine. $\mu$ TCA may also be a platform for a direct sampling sub-system. The basic features are: support of high accuracy (up to 16 bit) and high speed (up to 1Gsps) ADCs. An application specific analog part of the sub-system is positioned on FPC mezzanines (FPGA mezzanine card standard ANSI/VITA 57.1). Common components like FPGA, power supplies, logics, management, etc., are placed on the base board. A lot of commercial ready FMC modules, featuring high performance FPGA circuits, are available with a variety of signal processing functions. FMC minimizes the signal latency while maximizing the data throughput. Dedicated FMC modules are designed for specific purposes and then are reused in other of future projects. $\mu \mathrm{TCA}$ carrier boards support double width or two single width FMC. They display AMC.4 compatibility with IPMI support. AMC.4 and FMC I/Os include: PPC CPU, GEth, PCIe, P2P links, M-LVDS, CLKA and CLKB routed to FPGA, JTAG, many TP links, USB. FMC boards serve customary tasks including analog signal processing (analog mezzanines). The ATCA and /or $\mu$ TCA crates with versatile RTM (Rear Transaction Modules) serve the general purposes of making the whole system work. The system was built inside the EuCARD project.

\section{Conclusions, ACKnowledgments, Future}

WILGA Symposium from its beginning in the mid nineties has produced more than 2500 articles, out of which over 1000 were published in Proc.SPIE. Several hundred of them are associated with the research activities of the PERG/ELHEP Research Group at ISE WUT. The Group is an initiator and major organizer of the WILGA Symposia.

The WILGA 2012 meeting was a fruitful event gathering young researchers from the fields of photonics and electronics systems. Photonics and Web Engineering 2012, in its $30^{\text {th }}$ edition keeps the style of being a wide and friendly meeting for young researchers, mainly Ph.D. students. The Wilga Symposium proceedings are good memoirs of the development of photonics, optoelectronics, metrology and some telecom technologies in the academic laboratories in this country.

The author would like to acknowledge all the persons involved in the organization of the conference on 'Photonics and Web Engineering', Wilga 2012, in particular the Program and Organization Committees. The author also would like to thank all participants of Wilga Symposium for making the event again and again a success. This paper was prepared using the invited and contributed presentations debated during Wilga 2012. Some fragments of the text were quoted from these presentations and from session discussions.

The 2013 Symposia on Photonics and Internet Engineering will be held on 26-29 January at WEiTI PW building in Warsaw and on 28.05-03.06 in Wilga Resort by PW. The organizers warmly invite young researchers to present their work. The WILGA Symposium web page is: http://wilga.ise.pw.edu.pl.

\section{REFERENCES}

[1] R. Romaniuk, "Manufacturing and characterization of ring-index optical fibers," Optica Applicata, vol. 31, no. 2, pp. 425-444, 2001.

[2] R. Romaniuk et al., "Multicore single-mode soft-glass optical fibers," Optica Applicata, vol. 29, no. 1, pp. 15-49, 1999.
[3] J. Dorosz and R. Romaniuk, "Fiber Optics Department of Biaglass Co. twenty years of research activities," Optica Applicata, vol. 28, no. 4, pp. 267-291, 1998.

[4] - "Multicrucible technology of tailored optical fibres," Optica Applicata, vol. 28, no. 4, pp. 293-322, 1998.

[5] R.Romaniuk, "Tensile strength of tailored optical fibres," Optoelectronics Review, vol. 8, no. 2, pp. 101-116, 2000.

[6] R. Romaniuk, "Capillary optical fiber - design, fabrication, characterization and application," Bulletin of the Polish Academy of Sciences: Technical Sciences, vol. 56, no. 2, pp. 87-102, 2008.

[7] R. Romaniuk, K. Pozniak, T. Czarski, and G. Kasprowicz et al., "Optical network and fpga/dsp based control system for free electon laser," Bulletin of the Polish Academy of Sciences: Technical Sciences, vol. 53, no. 2, pp. 123-138, 2005.

[8] A. Dybko, R. Romaniuk, W. Wróblewski, and Z. Brzózka et al., "Assessment of water quality based on multiparmeter fiber optic probe," Sensors and Actuators, B: Chemical, vol. 51, no. 1-3, pp. 208-213, 1998.

[9] — , "Efficient reagent immobilization procedure for ion-sensitive optomembranes," Sensors and Actuators, B: Chemical, vol. 39, no. 1-3, pp. 207-211, 1997.

[10] — "Applications of optical fibres in oxidation-reduction titrations," Sensors and Actuators, B: Chemical, vol. 29, no. 1-3, pp. 374-377, 1995.

[11] _ _ "Polymer track membranes as a trap support for reagent in fiber optic sensors," Journal of Applied Polymer Sciences, vol. 59, no. 4, pp. 719-723, 1996.

[12] B. Mukherjee, D. Rybka, and R. Romaniuk et al., "Application of low-cost Gallium Arsenide light-mitting-diodes as kerma dosemeter and fluence monitor for high-energy neutrons," Radiation Protection Dosimetry, vol. 126, no. 1-4, pp. 256-260, 2007.

[13] R. Romaniuk and K. Pozniak, "Metrological aspects of accelerator technology and high energy physics experiments," Measurement Science and Technology, vol. 18, no. 8, 2008, art. no. E01.

[14] P. Fafara, W. Jalmuzna, W. Koprek, R. Romaniuk, and K. Pozniak et al., "FPGA-based implementation of a cavity field controller for FLASH and X-FEL," Measurement Science and Technology, vol. 18, no. 8, pp. 2365-2371, 2008

[15] A. Burd, M. Cwiok, M. Husejko, and R. Romaniuk et al., "Pi of the sky - all-sky, real-time search for fast optical transients," New Astronomy, vol. 10 , no. 5, pp. 409-416, 2005.

[16] A. Burd, K. T. Pozniak, and R. S. Romaniuk et al., "Pi of the sky' - automated search for fast optical transients over the whole sky," Astronomische Nachrichten, vol. 325, no. 6-8, p. 674, 2004.

[17] W. Ackerman, K. Pozniak, R. Romaniuk, and P. Pucyket al., "Operation of a free-electron laser from the extreme ultraviolet to the water window," Nature Photonics, vol. 1, no. 6, pp. 336-342, 2007.

[18] T. Czarski, K. T. Pozniak, and R. S. Romaniuk et al., "Superconducting cavity driving with FPGA controller," Nuclear Instruments and Methods in Physics Research A, vol. 568, no. 2, pp. 854-862, 2006.

[19] _ "TESLA cavity modeling and digital implementation in FPGA technology for control system development," Nuclear Instruments and Methods in Physics Research A, vol. 556, no. 2, pp. 565-576, 2006.

[20] - "Cavity parameters identification for TESLA control system development," Nuclear Instruments and Methods in Physics Research A, vol. 548, no. 3, pp. 283-297, 2005.

[21] R. S. Romaniuk, "Petabit photonic internet," Photonics Letters of Poland, vol. 3, no. 2, pp. 91-93, 2011.

[22] P. Obroślak, K. T. Pozniak, and R. S. Romaniuk et al., "Digital techniques for noise reduction in CCD detectors," Photonics Letters of Poland, vol. 2, no. 3, pp. 134-136, 2010.

[23] R. Romaniuk, "Wilga photonics and web engineering 2010," Photonics Letters of Poland, vol. 2, no. 2, pp. 55-57, 2010.

[24] _ " "Geometry design in refractive capillary optical fibers," Photonics Letters of Poland, vol. 2, no. 2, pp. 64-66, 2010.

[25] _ "Modal structure design in refractive capillary optical fibers," Photonics Letters of Poland, vol. 2, no. 1, pp. 22-24, 2010.

[26] — " "The Photonics Letter of Poland' A new peer-reviewed internet publication of the Photonics Society of Poland," Photonics Letters of Poland, vol. 1, no. 1, pp. 1-3, 2009.

[27] G. Kasprowicz et al., "CCD detectors for wide field optical astronomy,' Photonics Letters of Poland, vol. 1, no. 2, pp. 82-84, 2009.

[28] R. S. Romaniuk, "Wilga symposium on photonics applications," Photonics Letters of Poland, vol. 1, no. 2, pp. 46-48, 2009.

[29] R. Romaniuk, "POLFEL - A free electron laser in Poland," Photonics Letters of Poland, vol. 1, no. 3, pp. 103-105, 2009. 
[30] T. R. Wolinski and R. Romaniuk, "Photonics Society of Poland established," Metrology and Measurement Systems, vol. 15, no. 2, pp. 241245, 2008.

[31] R. Romaniuk, "Search for ultimate throughput in ultra-broadband photonic Internet," International Journal of Electronics and Telecommunications, vol. 57, no. 4, pp. 523-528, 2011.

[32] _ - "Photonics and web engineering 2011," International Journal of Electronics and Telecommunications, vol. 57, no. 3, pp. 421-428, 2011

[33] — "Accelerator infrastructure in Europe EuCARD 2011" Interna tional Journal of Electronics and Telecommunications, vol. 57, no. 3, pp. 413-419, 2011.

[34] J. Dorosz and R. Romaniuk, "Development of optical fiber technology in Poland," International Journal of Electronics and Telecommunications, vol. 57, no. 2, pp. 191-197, 2011.

[35] R. Romaniuk, "Advanced photonic and electronic systems Wilga 2010," International Journal of Electronics and Telecommunications, vol. 56, no. 4, pp. 479-484, 2010.

[36] —_ "EuCARD 2010 accelerator technology in Europe," International Journal of Electronics and Telecommunications, vol. 56, no. 4, pp. 485 488,2010

[37] W. Wojcik and R. Romaniuk, "Development of optical fiber technology in Poland," International Journal of Electronics and Telecommunications, vol. 56, no. 1, pp. 99-104, 2010.

[38] S. Chatrchyan, K. Pozniak, R. Romaniuk, and W. Zabolotny, "The CMS Collaboration, et al., The CMS experiment at the CERN LHC," Journal of Instrumentation, vol. 3, no. 8, 2008, art. no. S08004.

[39] - "The CMS Collaboration, et al., Commissioning of the CMS experiment and the cosmic run at four tesla," Journal of Instrumentation, vol. 5, no. 3, 2010, art. no. T03001.

[40] R. S. Romaniuk, "Wilga Photonics Applications and Web Engineering,' Proceedings of SPIE, vol. 8454, January 2012, art. no. 845402.

[41] _ "Astronomy and Space Technologies, Photonics Applications and Web Engineering, Wilga May 2012," Proceedings of SPIE, vol. 8454 2012, art. no. 845403

[42] —-, "Accelerator Technology and High Energy Physics Experiments, Photonics Applications and Web Engineering, Wilga May 2012," Proceedings of SPIE, vol. 8454, 2012, art. no. 845404.

[43] _ "Photon Physics and Plasma Research, Photonics Applications and Web Engineering, Wilga May 2012,' Proceedings of SPIE, vol. 8454 2012, art. no. 845405 .

[44] - "Optoelectronic Devices, Sensors, Communication and Multimedia, Photonics Applications and Web Engineering, Wilga May 2012,' Proceedings of SPIE, vol. 8454, 2012, art. no. 845406.
[45] — "Biomedical, Artificial Intelligence and DNA Computing, Photonics Applications and Web Engineering, Wilga May 2012," Proceedings of SPIE, vol. 8454, 2012, art. no. 845407.

[46] — "Accelerator Science and Technology in Europe - EuCARD 2012," Proceedings of SPIE, vol. 8454, 2012, art. no. 845400

[47] B. Niton, K. T. Pozniak, and R. S. Romaniuk, "A plug-in to eclipse for VHDL source codes: functionalities," Proceedings of SPIE, vol. 8454, 2012, art. no. $84542 \mathrm{C}$

[48] A. Zagozdzinska, K. T. Pozniak, and R. S. Romaniuk, "Heavy stable charged particles search by novel pattern comparator," Proceedings of SPIE, vol. 8454, 2012, art. no. 84540P.

[49] J. R. Just et al., "Highly parallel distributed computing system with optical interconnections," Microprocessing and Microprogramming, vol. 27, no. 1-5, pp. 489-493, 1989.

[50] R. S. Romaniuk, "Multicore optical fibres," Revue Roumaine de Physique, vol. 32, no. 1-2, pp. 99-112, 1987.

[51] I. U. Romaniuk and R. Romaniuk, "Light-conducting-fibre properties of retinal receptors," Klinika Oczna, vol. 83, no. 1, pp. 29-30, 1981.

[52] — - "The use of light-conducting fibres in ophthalmological equipment," Klinika Oczna, vol. 83, no. 1, pp. 31-33, 1981

[53] R. S. Romaniuk, "Polish Debut," Photonics Spectra, vol. 26, no. 5, pp. 10-12, May 1992.

[54] — "The photonics scene in the new Poland," Photonics Spectra, vol. 26, no. 4, pp. 64-65, April 1992.

[55] W. Zabolotny, P. Roszkowski, K. Kierzkowski, K. Pozniak, and R. Romaniuk et al., "Distributed embedded PC based control and data acquisition system for Tesla cavity controller and simulator," Proceedings of SPIE, vol. 5484, pp. 171-179, 2004.

[56] R. S. Romaniuk, "Photonics and Intenret Engineering," Elektronika, konstrukcje technologie zastosowania, vol. 52, no. 3, pp. 163-164, 2011 , (in Polish).

[57] — "Ultra broadband photonic Internet," Proceedings of SPIE, vol. 8010, 2011, art. no. 801003

[58] J. Modelski and R. S. Romaniuk "Electronics and telecommunications in Poland, issues and perspectives, Part I: Society and education," Proceedings of SPIE, vol. 7745, 2010, art. no. 774504

[59] _ "Electronics and telecommunications in Poland, issues and perspectives, Part II: Science, research, development, higher education,' Proceedings of SPIE, vol. 7745, 2010, art. no. 774505

[60] - "Electronics and telecommunications in Poland, issues and perspectives, Part III: Innovativeness, applications, economy, development scenarios, politics," Proceedings of SPIE, vol. 7745, 2010, art. no. 774506. 